\title{
CONCERNING TOPONOGOV'S THEOREM AND LOGARITHMIC IMPROVEMENT OF ESTIMATES OF EIGENFUNCTIONS
}

\author{
MATTHEW D. BLAIR AND CHRISTOPHER D. SOGGE
}

\begin{abstract}
We use Toponogov's triangle comparison theorem from Riemannian geometry along with quantitative scale oriented variants of classical propagation of singularities arguments to obtain logarithmic improvements of the Kakeya-Nikodym norms introduced in 21. for manifolds of nonpositive sectional curvature. Using these and results from our paper 4 we are able to obtain log-improvements of $L^{p}(M)$ estimates for such manifolds when $2<p<\frac{2(n+1)}{n-1}$. These in turn imply $(\log \lambda)^{\sigma_{n}}$, $\sigma_{n} \approx n$, improved lower bounds for $L^{1}$-norms of eigenfunctions of the estimates of the second author and Zelditch [25, and using a result from Hezari and the second author [17, under this curvature assumption, we are able to improve the lower bounds for the size of nodal sets of Colding and Minicozzi [1] by a factor of $(\log \lambda)^{\mu}$ for any $\mu<\frac{2(n+1)^{2}}{n-1}$, if $n \geq 3$.
\end{abstract}

\section{Introduction.}

The purpose of this paper is to show that if $(M, g)$ is a compact Riemannian manifold of dimension $n \geq 2$ then one obtains logarithmically improved Kakeya-Nikodym bounds of eigenfunctions and appropriate eigenfunctions in all dimensions, as well as log-improved restriction estimates in two-dimensions. Using results from a companion paper [4, we deduce that we have improved $L^{p}(M)$ estimates for the range $2<p<\frac{2(n+1)}{n-1}$. These imply log-improvements of the $L^{1}(M)$ bounds of the second author and Zelditch [25], which in turn imply log-improvements (assuming nonpositive curvature) of the lower bounds of Colding and Minicozzi [11 for the size of nodal sets. The assumption of nonpositive curvature is needed since, except for the last estimate regarding nodal sets, all of the estimates are saturated by the highest weight spherical harmonics, on $S^{n-1}$, $k^{\frac{n-1}{4}} \operatorname{Re}\left(x_{1}+i x_{2}\right)^{k}, k=1,2, \ldots$ They also are saturated by the Gaussian beams constructed by Ralston [18], which cannot exist on manifolds of nonpositive curvature.

We shall consider $L^{2}$-normalized eigenfunctions on a Riemannian manifold $(M, g)$ of dimension $n \geq 2$. So we are considering functions satisfying

$$
\left(\Delta_{g}+\lambda^{2}\right) e_{\lambda}=0, \quad \text { and } \int_{M}\left|e_{\lambda}\right|^{2} d V_{g}=1,
$$

where $\Delta_{g}$ and $d V_{g}$ of course are the Laplace-Beltrami operator and volume element associated with the metric $g$ on $M$, respectively. So in our case $\lambda$ is the frequency of the eigenvalue, and also $e_{\lambda}$ is an eigenfunction with eigenvalue $\lambda$ of $P=\sqrt{-\Delta_{g}}$.

2010 Mathematics Subject Classification. Primary 58J51; Secondary 35A99, 42B37.

Key words and phrases. Eigenfunctions, Kakeya-Nikodym averages.

The authors were supported in part by the NSF grants DMS-1301717 and DMS-1361476, respectively. 
In what follows, we let $\Pi$ denote the space of all geodesic segments of length one, assuming that Inj $M \geq 10$, where Inj $M$ denotes the injectivity radius of $(M, g)$. Otherwise, we shall modify things so that the geodesics in $\Pi$ are of length Inj $M / 10$. This slight ambiguity is caused by the fact that we shall want to assume eventually that the sectional curvatures of $(M, g)$ are pinched below by -1 to simplify the statement of the cone comparison result that we shall employ, which play a critical role in our analysis.

If $\gamma \in \Pi, \mathcal{T}_{\varepsilon}(\gamma)$ denotes a geodesic tube of width $\varepsilon$ about $\gamma \in \Pi$, then the KakeyaNikodym norm of our eigenfunctions defined by

$$
\left\|e_{\lambda} \mid\right\|_{K N}=\left(\sup _{\gamma \in \Pi} \int_{\mathcal{T}_{\lambda-\frac{1}{2}}(\gamma)}\left|e_{\lambda}\right|^{2} d V_{g}\right)^{\frac{1}{2}},
$$

were introduced by one of us in [21], following earlier related work of Bourgain [5], as a way of controlling the $L^{p}(M)$ norms of eigenfunctions. Although not explicitly stated, the inequalities proved in [21] yield

$$
\left\|e_{\lambda}\right\|_{L^{4}(M)} \leq C \lambda^{\frac{1}{8}}\left\|e_{\lambda}\right\|_{K N}^{\frac{1}{4}}, \quad \text { if } n=2,
$$

and hence improvements over the trivial estimate

$$
\left\|e_{\lambda} \mid\right\|_{K N} \leq 1
$$

would yield improvements over the second author's earlier bounds [19] $\left\|e_{\lambda}\right\|_{4}=O\left(\lambda^{\frac{1}{8}}\right)$ (saturated on $S^{2}$ ). By interpolating with the $L^{6}$ estimate there, one would also get improvements for the full range $2<p<6$. The trivial Kakeya-Nikodym bounds were improved by the second author and Zelditch in [27, who showed that, in two dimensions, one has $\left\|e_{\lambda}\right\| \|_{K N}=o(1)$, and, as a result, the improvement $\left\|e_{\lambda}\right\|_{4}=o\left(\lambda^{\frac{1}{8}}\right)$, under the assumption of nonpositive curvature. These sorts of results were extended to higher dimensions by the authors in [2]. In all cases, though, even though we could show that the various norms were relatively small as $\lambda \rightarrow \infty$, there was no control on the rate of decay of the Kakeya-Nikodym norms or on the way that the constants in the $L^{p}$ improvements over the ones in [19] go to zero as $\lambda \rightarrow \infty$.

The purpose of this paper is to establish that there are improvements in turns of powers of $\log \lambda$ for all of these things. Our main result, which along with estimates in a companion paper, yields these improved bounds.

Theorem 1.1. Suppose $(M, g)$ has nonpositive sectional curvatures. Then

$$
\sup _{\gamma \in \Pi} \int_{\mathcal{T}_{\lambda}-\frac{1}{2}}(\gamma)=\left.e_{\lambda}\right|^{2} d V \lesssim c(\lambda)
$$

for $\lambda \gg 1$ with

$$
c(\lambda)=\left\{\begin{array}{l}
(\log \lambda)^{-\frac{1}{2}}, \quad \text { if } n=2 \\
(\log \lambda)^{-1} \log \log \lambda, \quad \text { if } n=3 \\
(\log \lambda)^{-1}, \quad \text { if } n \geq 4
\end{array}\right.
$$

Moreover, if $n=2$, we have

$$
\sup _{\gamma \in \Pi} \int_{\gamma}\left|e_{\lambda}\right|^{2} d s \leq C \lambda^{\frac{1}{2}} c(\lambda)
$$


The above estimates hold as well when $e_{\lambda}$ is replaced by a quasi-mode satisfying

$$
\left\|\psi_{\lambda}\right\|_{L^{2}(M)}+(\log \lambda / \lambda)\left\|\left(\Delta_{g}+\lambda^{2}\right) \psi_{\lambda}\right\|_{L^{2}(M)} \leq 1 .
$$

The estimate $(1.4)$ is stronger than $(1.3)$ when $n=2$. Moreover, it represents a logarithmic improvement over the two-dimensional restriction estimates of Burq, Gérard and Tzvetkov [6]. As we pointed out in [2] in higher dimensions, $n \geq 4$, restriction estimates, as opposed to the Kakeya-Nikodym tube estimates as in $[1.3)$, are too singular to control $L^{p}$ norms. In these dimensions they are saturated by eigenfunctions matching the profile of zonal spherical harmonics, rather than highest weight spherical harmonics which saturate the Kakeya-Nikodym norms in 1.3 . In this case, it is also a bit more straightforward to obtain logarithmic improvements over the geodesic restriction estimates in [6]. This was done by Chen [9] when $n>3$. Chen and one of us [10] also showed that when $n=3$ one could improve on the universal bounds (saturated in this case by both zonal harmonics and highest weight spherical harmonics) assuming that $(M, g)$ is of constant nonpositive curvature. Whether one can get logarithmic improvements for either constant or variable nonpositive curvature is an interesting open problem in this dimension.

The proof of these results will follow closely the general scheme introduced in [21] and 27]. The new ingredient is that we are using classical triangle comparison theorems from Riemannian geometry to make tighter pseudo-differential cutoffs allowing us to use the time-averaging method over logarithmic time intervals (as opposed to large ones basically not depending on $\lambda)$. We use the universal cover of $(M, g)$ to break up the operators that we use to obtain our estimates into a number of pieces. We use quantitative and scale oriented variants of classical propagation of singularities arguments (i.e., integration by parts) to handle what turn out to be the small, but numerous, "error" terms which arise from terms associated with a portion of the universal cover not contained in a natural cone of small apeture about the geodesic. We can handle the relatively few remaining "local terms" in standard ways.

Before we turn to the proof of Theorem let us state a couple of corollaries of our main theorem and results from our companion paper 44. The first concerns logarithmically improved $L^{p}$ norms of eigenfunctions and appropriate quasi-modes.

Corollary 1.2. Assume, as above, that $(M, g)$ is a compact $n \geq 2$ dimensional manifold with nonpositive sectional curvatures. Then for any $2<p<\frac{2(n+1)}{n-1}$ there is a number $\sigma(p, n)>0$ so that

$$
\left\|e_{\lambda}\right\|_{L^{p}(M)} \lesssim \lambda^{\frac{n-1}{2}\left(\frac{1}{2}-\frac{1}{p}\right)}(\log \lambda)^{-\sigma(p, n)} .
$$

Furthermore, if $\frac{2(n+2)}{n}<p<\frac{2(n+1)}{n-1}$, one can take

$$
\sigma(p, n)=\left\{\begin{array}{l}
\frac{n+1}{n-1}\left(\frac{1}{p}-\frac{n-1}{2(n+1)}\right), \text { if } n \geq 4, \\
\text { any } \sigma(p, 3)<2\left(\frac{1}{p}-\frac{1}{4}\right), \text { if } n=3, \\
\frac{3}{2}\left(\frac{1}{p}-\frac{1}{6}\right), \text { if } n=2 .
\end{array}\right.
$$

The above estimates hold as well when $e_{\lambda}$ is replaced by a quasi-mode satisfying 1.5. 
Let us show how we can obtain these $L^{p}$ norms for eigenfunctions and postpone the discussion of quasi-modes for a bit. To prove 1.6 we shall use (1.3) and the following estimate from our companion paper [4,

$$
\left\|e_{\lambda}\right\|_{L^{p}(M)} \lesssim \lambda^{\frac{n-1}{2}\left(\frac{1}{2}-\frac{1}{p}\right)}\left\|e_{\lambda}\right\|_{K N}^{\frac{2(n+1)}{n-1}\left(\frac{1}{p}-\frac{n-1}{2(n+1)}\right)}, \quad \text { if } \quad \frac{2(n+2)}{n}<p<\frac{2(n+1)}{n-1} .
$$

Using this estimate and our Kakeya-Nikodym bounds 1.3 we immediately get 1.6 for the range $\frac{2(n+2)}{n}<p<\frac{2(n+1)}{n-1}$, and the estimates from the remaining range $2<p \leq$ $\frac{2(n+2)}{n}$ follow from interpolation with the trivial bound $\left\|e_{\lambda}\right\|_{2} \leq 1$.

Using these estimates we can in turn get improvements for lower bounds of the $L^{1}$ norms of eigenfunctions and the size of their nodal sets under our curvature assumptions:

Corollary 1.3. Assume that $(M, g)$ is as above. Then

$$
\lambda^{-\frac{n-1}{4}}(\log \lambda)^{\mu} \lesssim\left\|e_{\lambda}\right\|_{L^{1}(M)}
$$

for any $\mu<\mu_{n}$ with

$$
\mu_{n}= \begin{cases}\frac{(n+1)^{2}}{n-1}, & \text { if } n \geq 3 \\ \frac{(n+1)^{2}}{2(n-1)}, & \text { if } n=2 .\end{cases}
$$

Consequently, if $e_{\lambda}$ is a real-valued eigenfunction and $\left|Z_{\lambda}\right|$ denotes the $(n-1)$-dimensional Hausdorff measure of its nodal set, $Z_{\lambda}=\left\{x: e_{\lambda}(x)=0\right\}$, we have

$$
\lambda^{1-\frac{n-1}{2}}(\log \lambda)^{2 \mu} \lesssim\left|Z_{\lambda}\right|
$$

when $\mu<\mu_{n}$. In particular, when $n=3,(\log \lambda)^{r} \lesssim\left|Z_{\lambda}\right|$ for all $r<16$.

The lower bound of $\lambda^{1-\frac{n-1}{2}} \lesssim\left|Z_{\lambda}\right|$ is due to Colding and Minicozzi [1] and it is the best known lower bound for general $C^{\infty}$ manifolds. An alternate proof of this lower bound was later found by the author and Zelditch [26]. In the real analytic case Donnelly and Fefferman 12 showed that $\left|Z_{\lambda}\right| \approx \lambda$. Earlier Yau 33 had conjectured this bound for general smooth Riemannian manifolds.

To prove 1.9, we first notice that, by Hölder's inequality

$$
1=\left\|e_{\lambda}\right\|_{L^{2}(M)} \leq\left\|e_{\lambda}\right\|_{L^{1}(M)}^{\frac{p-2}{2(p-1)}}\left\|e_{\lambda}\right\|_{L^{p}(M)}^{\frac{p}{2(p-1)}}
$$

and so

$$
\lambda^{-\frac{n-1}{4}}\left(\lambda^{-\frac{n-1}{2}\left(\frac{1}{2}-\frac{1}{p}\right)}\left\|e_{\lambda}\right\|_{L^{p}(M)}\right)^{-\frac{p}{p-2}} \leq\left\|e_{\lambda}\right\|_{L^{1}(M)}
$$

To get 1.9 we plug the estimates 1.6 into this inequality and realize that the resulting lower bounds improve as $p \searrow \frac{2(n+2)}{n}$. The power $\mu_{n}$ represents what we would obtain if (1.6) were valid at the endpoint, which thus gives us 1.9 . To prove 1.10 we just use the lower bound of Hezari and the second author [17,

$$
\lambda\left\|e_{\lambda}\right\|_{L^{1}(M)}^{2} \lesssim\left|Z_{\lambda}\right|,
$$

which was proved using ideas from an earlier work of the second author and Zelditch [25].

In a recent work, Hezari and Rivière [16] were able to obtain log-improvements for $L^{p}$-norms of a subsequence of eigenfunctions of density one assuming that the sectional curvatures of $(M, g)$ are strictly negative. Our results relax the latter condition to our 
assumption that the curvatures be nonpositive, but, more significantly, we are able to handle all eigenfunctions and not just ones corresponding to a density one subsequence of eigenvalues. The results of Hezari and Rivière [16] are based on obtaining non-trivial log-improvements of $L^{2}$-norms of eigenfunctions over shrinking balls (which had also been obtained earlier and independently by Han [14]) and then using an estimate that relates such estimates to $L^{p}$-norms (see also [22] for the latter). Over the years, there have been

many works on improving $L^{p}$-norms for relatively large exponents $p>\frac{2(n+1)}{n-1}$, including [15], 23], 24], 28] and [29. Hassell and Tacy [15] extended Bérard's sup-norm estimate, implicit in [1, by showing that there are $(\log \lambda)^{-1 / 2}$ improvements over the bounds of [19] under the assumption of nonpositive curvature for all $p>\frac{2(n+1)}{n-1}$. The endpoint case where $p=\frac{2(n+1)}{n-1}$ is the remaining case where general results concerning improvements for the full sequence of eigenfunctions under natural geometric assumptions remains open.

\section{Some reductions and tools.}

To prove 1.3 , we shall use the fact that if $\rho \in \mathcal{S}(\mathbb{R})$ satisfies

$$
\rho(0)=1 \quad \operatorname{supp} \hat{\rho} \subset[-1 / 2,1 / 2],
$$

then

$$
\rho(T(\lambda-P)) e_{\lambda}=e_{\lambda} \text { if } P=\sqrt{-\Delta_{g}} .
$$

Consequently, we would have the estimate $(1.3)$ for eigenfunctions if we could show that we have

$$
\left.\|\rho(T(\lambda-P)) f\|_{L^{2}\left(\mathcal{T}_{\lambda}-\frac{1}{2}\right.}(\gamma)\right) \lesssim \sqrt{c(\lambda)}\|f\|_{L^{2}(M)}, \quad T \approx \log \lambda
$$

where the constants involved are independent of $\gamma \in \Pi$.

Let us also see why (2.3) implies that (1.3) also holds for quasi-modes satisfying (1.5). Let $\left\{e_{j}\right\}$ denote an orthonormal basis of eigenfunctions with eigenvalues $\lambda_{j} \rightarrow \infty$ and let $E_{j}$ denote the projection onto the $j$ th eigenspace. Then since $\rho(0)=1$ and since $T \approx \log \lambda$, it is not difficult to see that if we define the spectral projectors associated to windows of width $(\log \lambda)^{-1}$, i.e.,

$$
E_{\left[\lambda, \lambda+(\log \lambda)^{-1}\right]} f=\sum_{\lambda_{j} \in\left[\lambda, \lambda+(\log \lambda)^{-1}\right]} E_{j} f,
$$

we have

$$
\left.\left\|E_{\left[\lambda, \lambda+(\log \lambda)^{-1}\right]} f\right\|_{L^{2}(\mathcal{T}}{ }_{\lambda-\frac{1}{2}}(\gamma)\right) \lesssim \sqrt{c(\lambda)}\|f\|_{L^{2}(M)}
$$

Using this it is very simple to deduce that 1.3 also must hold for functions $\psi_{\lambda}$ satisfying 1.5]. (See 30] for similar arguments.)

The $L^{p}$ estimates for quasi-modes are proved by a similar argument. Theorem 1.1 in 4. shows that for $T$ as above

$$
\begin{aligned}
\|\rho(T(\lambda-P)) f\|_{L^{p}(M)} \lesssim \lambda^{\frac{n-1}{2}\left(\frac{1}{2}-\frac{1}{p}\right)}\|\rho(T(\lambda-P)) f\|_{K N}^{\frac{2(n+1)}{n-1}\left(\frac{1}{p}-\frac{n-1}{2(n+1)}\right)}, \\
\quad \text { if } \frac{2(n+2)}{n}<p<\frac{2(n+1)}{n-1} .
\end{aligned}
$$


If we combine this with (2.3), we deduce that for exponents such exponents we have

$$
\|\rho(T(\lambda-P)) f\|_{L^{p}(M)} \lesssim \lambda^{\frac{n-1}{2}\left(\frac{1}{2}-\frac{1}{p}\right)}(\log \lambda)^{-\sigma(p, n)}\|f\|_{L^{2}(M)} .
$$

Since $T \approx \log \lambda$ and $\rho(0)=1$, this in turn implies that

$$
\left\|E_{\left[\lambda, \lambda+(\log \lambda)^{-1}\right]} f\right\|_{L^{p}(M)} \lesssim \lambda^{\frac{n-1}{2}\left(\frac{1}{2}-\frac{1}{p}\right)}(\log \lambda)^{-\sigma(p, n)}\|f\|_{L^{2}(M)} .
$$

By interpolating with the trivial $L^{2}$ estimate we see that we also get bounds of this

type for $2<p \leq \frac{2(n+2)}{n}$. These $L^{2} \rightarrow L^{p}$ bounds for $(\log \lambda)^{-1}$ sized spectral projector operators are easily seen to imply that (1.6) is also valid for quasi-modes satisfying 1.5 (see [30]).

To prove 2.3), we note that this is equivalent to showing that

$$
\begin{aligned}
& \|\chi(T(\lambda-P)) f\|_{L^{2}\left(\mathcal{T}_{\lambda^{-\frac{1}{2}}}(\gamma)\right)} \lesssim c(\lambda)\|f\|_{L^{2}(M)}, \\
& \quad \text { if } \operatorname{supp} f \subset \mathcal{T}_{\lambda^{-\frac{1}{2}}}(\gamma) \text { and } \quad T \approx \log \lambda,
\end{aligned}
$$

with $\chi=|\rho|^{2}$. We note then that

$$
\widehat{\chi} \subset[-1,1] .
$$

We shall take $T$ to be $c \log \lambda$ where $c>0$ will be a small constant depending on a lower bound for the sectional curvatures of $(M, g)$, among other things. There is no loss of generality in assuming, as we shall, that they satisfy

$$
K \geq-1,
$$

and we also recall that we are assuming that they are everywhere nonpositive.

We shall then use quantitative microlocal analysis bounds and the following geometric fact, which is a consequence of Toponogov's triangle comparison theorem, to prove Theorem 1.1

Proposition 2.1 (Toponogov). Equip $\mathbb{R}^{n}$ with a metric $\tilde{g}$ of nonpositive curvature and assume that the sectional curvatures, $K$, of $\left(\mathbb{R}^{n}, \tilde{g}\right)$ also satisfy

$$
K \geq-1 .
$$

Let $\tilde{\gamma}(t), t \in \mathbb{R}$, be a geodesic with $\tilde{\gamma}(0)=P$. Given $T \gg 1$, let $C(\theta ; T), \theta \ll 1$, denote the set of points $Q \in B_{\tilde{g}}(P ; T)$ which lie on a geodesic though $P$ which intersects $\tilde{\gamma}$ of angle $\leq \theta$. Thus, $C(\theta ; T)$ is the intersection of the geodesic ball $B_{\tilde{g}}(P ; T)$ with the cone of aperture $\theta$ about $\tilde{\gamma}$ with vertex $P$. Fix $R>0$. Then if

$$
\mathcal{T}_{R}(\tilde{\gamma})=\left\{x \in \mathbb{R}^{n}: d_{\tilde{g}}(x, \tilde{\gamma}) \leq R\right\}
$$

we have that

$$
C\left(\theta_{T} ; T\right) \subset \mathcal{T}_{R}(\tilde{\gamma}), \quad \text { if } \quad \sin \frac{1}{2} \theta_{T}=\frac{\sinh \frac{1}{2} R}{\sinh T}, \quad \text { if } T>0
$$

Remark: To simplify the notation we are assuming throughout that the nonpositive curvature is pinched below by -1 . If we assumed the nonpositive sectional curvatures 
were bounded below by $-\kappa^{2}$, then the proof of Proposition 2.1 which we shall present also gives that

$$
C\left(\theta_{T, \kappa} ; T\right) \subset \mathcal{T}_{R}(\tilde{\gamma}), \quad \text { if } \sin \frac{1}{2} \theta_{T, \kappa}=\frac{\sinh \frac{\kappa}{2} R}{\sinh \kappa T}, \quad \text { if } T>0 .
$$

Sending $\kappa \searrow 0$, despite being slightly weaker, one essentially recovers the familiar "sine equals opposite over hypothesis" in Euclidean geometry.

The other main tool that we shall use, as in our earlier related works [27, 4], is a scale oriented microlocalization about the unit cosphere bundle $S^{*} \gamma \subset S^{*} M$ associated with our $\gamma \in \Pi$. We may work in local coordinates so that $\gamma$ is just

$$
\{(t, 0, \ldots, 0): 0 \leq t \leq 1\} .
$$

Fix then $\alpha \in C_{0}^{\infty}(\mathbb{R})$ satisfying $\alpha(s)=1$ for $|s| \leq 1$ and $\alpha(s)=0$ for $|s| \geq 2$ and define compound symbols

$$
\begin{array}{r}
Q_{\theta, \lambda}(x, y, \xi)=\alpha\left(\theta^{-1} d_{g}(x, \gamma)\right) \alpha\left(\theta^{-1} d_{g}(y, \gamma)\right) \alpha\left(\theta^{-1}\left|\xi^{\prime}\right| /|\xi|\right) \Upsilon(|\xi| / \lambda), \\
\xi^{\prime}=\left(\xi_{2}, \ldots, \xi_{n}\right),
\end{array}
$$

where $\Upsilon \in C^{\infty}(\mathbb{R})$ is assumed to satisfy

$$
\Upsilon(s)=1, s \in\left[c_{0}, c_{0}^{-1}\right], \quad \Upsilon(s)=0, s \notin\left[\frac{c_{0}}{2}, 2 c_{0}^{-1}\right],
$$

with $c_{0}>0$ a small but fixed number to be specified later. We then define the associated integral operators $Q_{\theta, \lambda}$ with kernels

$$
Q_{\theta, \lambda}(x, y)=(2 \pi)^{-n} \int_{\mathbb{R}^{n}} e^{i\langle x-y, \xi\rangle} Q_{\theta, \lambda}(x, y, \xi) d \xi
$$

expressed in our local coordinates about $\gamma$.

In what follows we shall take $\lambda^{-\frac{1}{2}+\delta_{0}} \leq \theta \ll 1$ for some $\delta_{0}>0$ to also be specified later. Note that

$$
\begin{array}{r}
\left|D_{x, y}^{\alpha_{1}} D_{\xi}^{\alpha_{2}} Q_{\theta, \lambda}(x, y, \xi)\right| \leq C_{\alpha_{1}, \alpha_{2}} \theta^{-\left|\alpha_{1}\right|-\left|\alpha_{2}\right|} \lambda^{-\left|\alpha_{2}\right|}, \forall \alpha_{1}, \alpha_{2}, \\
\quad \text { and }\left|\partial_{\xi_{1}}^{j} Q_{\theta, \lambda}(x, y, \xi)\right| \leq C_{j} \lambda^{-j} .
\end{array}
$$

From this and a simple integration by parts argument we deduce that we have the uniform bounds for such $\theta$ and $\lambda \gg 1$

$$
\left|Q_{\theta, \lambda}(x, y)\right| \leq C_{N} \theta^{n-1} \lambda^{n}\left(1+\lambda\left|x_{1}-y_{1}\right|+\theta \lambda\left|x^{\prime}-y^{\prime}\right|\right)^{-N}, \quad \forall N=1,2, \ldots
$$

Consequently, we have the uniform bounds

$$
\begin{aligned}
& \sup _{x} \int\left|Q_{\theta, \lambda}(x, y)\right| d y, \sup _{y} \int\left|Q_{\theta, \lambda}(x, y)\right| d x \leq C, \\
& \quad \text { and }\left|Q_{\theta, \lambda}(x, y)\right| \leq C_{N} \theta^{n-1} \lambda^{n}\left(1+\theta \lambda d_{g}(x, y)\right)^{-N}, \quad N=1,2,3, \ldots
\end{aligned}
$$

We then shall use the following local result which is valid for all compact Riemannian manifolds, which also does not require the support assumptions in (2.5). It is based on the escape times of balls of radius $(\theta \lambda)^{-1}$ exiting $\mathcal{T}_{\lambda^{-\frac{1}{2}}}(\gamma)$ or $\gamma$ if they are at traveling at unit speed along geodesics of angle $\theta$ from $\gamma$. 
Proposition 2.2 (Escape times). Fix a compact Riemannian manifold $(M, g)$ of $d i$ mension $n \geq 2$, and let $a \in C_{0}^{\infty}((-1,1))$. Then if the constant $c_{0}>0$ in $\sqrt{2.9}$ is small enough

$$
\left\|\int a(t) e^{-i t \lambda}\left(I-Q_{\theta, \lambda}\right) e^{i t P} f d t\right\|_{L^{2}\left(\mathcal{T}_{\lambda^{-\frac{1}{2}}}(\gamma)\right)} \leq C_{\delta_{0}} \lambda^{-\frac{1}{4}} \theta^{-\frac{1}{2}}\|f\|_{L^{2}(M)},
$$

with $C_{\delta_{0}}$ independent of $\theta \geq \lambda^{-\frac{1}{2}+\delta_{0}}$, if $0<\delta_{0}<1 / 2$ is fixed and $\lambda$ is large. Moreover, when $n=2$ and $c_{0}>0$ is sufficiently small, we have, for any $\varepsilon>0$,

$$
\left\|\int a(t) e^{-i t \lambda}\left(I-Q_{\theta, \lambda}\right) e^{i t P} f d t\right\|_{L^{2}(\gamma)} \leq C_{\delta_{0}, \varepsilon} \theta^{-\frac{1}{2}-\varepsilon}\|f\|_{L^{2}(M)},
$$

with $L^{2}(\gamma)$ denoting the norm taken with respect to arc length measure over our $\gamma \in \Pi$. For a given $n$, the constants in 2.12) and (2.13) also only depend on the size of finitely many derivatives of $a$.

The two-dimensional estimate 2.13 is related to estimates of Greenleaf and Seeger [13] for Fourier integral operators associated to one-sided folding canonical relations and trace estimates for the wave equation of Tataru [31].

\section{The main argument.}

We shall postpone the proof of the two propositions until after this section. Now let us show how they imply our Kakeya-Nikodym estimates.

Let us split up our operators $\chi_{\lambda}$ into two pieces. The first,

$$
\chi_{\lambda}^{\theta}=Q_{\theta, \lambda} \circ \chi(T(\lambda-P))=\frac{1}{2 \pi T} \int_{-T}^{T} \widehat{\chi}(t / T) e^{-i \lambda t} Q_{\theta, \lambda} \circ e^{i t P} d t,
$$

denotes the microlocalization near the geodesic, which should be thought of as the "main" term, while the "remainder", $R_{\lambda}^{\theta}=\chi_{\lambda}-\chi_{\lambda}^{\theta}$, is given by

$$
R_{\lambda}^{\theta}=\left(I-Q_{\theta, \lambda}\right) \circ \chi(T(\lambda-P))=\frac{1}{2 \pi T} \int_{-T}^{T} \widehat{\chi}(t / T) e^{-i \lambda t}\left(I-Q_{\theta, \lambda}\right) \circ e^{i t P} d t .
$$

Using (2.12) it is very easy to handle the remainder on any manifold. No curvature assumptions are needed. Choose $a \in C_{0}^{\infty}(\mathbb{R})$ so that $\sum_{-\infty}^{\infty} a(t-k) \equiv 1$. Then since $e^{i k P}$ maps $L^{2}(M)$ to itself with norm 1, 2.12 implies that we have the uniform bounds

$$
\frac{1}{2 \pi T}\left\|\int a(t-k) \widehat{\chi}(t / T) e^{-i \lambda t}\left(I-Q_{\theta, \lambda}\right) e^{i t P} f d t\right\|_{L^{2}(M)} \leq C T^{-1} \lambda^{-\frac{1}{4}} \theta^{-\frac{1}{2}}\|f\|_{L^{2}(M)} .
$$

Since, in view of $\left(2.1^{\prime}\right)$ the left side is zero if $|k| \geq 2 T$, if we sum over these bounds we deduce that

$$
\left\|R_{\lambda}^{\theta} f\right\|_{L^{2}\left(\mathcal{T}-\frac{1}{2}(\gamma)\right)} \leq C \lambda^{-\frac{1}{4}} \theta^{-\frac{1}{2}}\|f\|_{L^{2}(M)} .
$$

We shall always assume that

$$
\theta \geq \lambda^{-\delta}, \quad \text { for some } 0<\delta \ll 1 / 2,
$$


which we can achieve by taking the $\delta_{0}$ in the Proposition 2.2 to be close to $1 / 2$. Consequently, if we assume that $\delta$ in $\sqrt{3.4}$ is small enough we get

$$
\left.\left\|R_{\lambda}^{\theta} f\right\|_{L^{2}\left(\mathcal{T}-\frac{1}{2}\right.}(\gamma)\right) \leq C \lambda^{-\frac{1}{8}}\|f\|_{L^{2}(M)}
$$

which is much better than the bounds posited in 2.5 . We note here that, in addition to making no curvature assumptions, we are not requiring the support assumptions in 2.5 .

On account of $(3.5)$, we would have 2.5 if we could show that

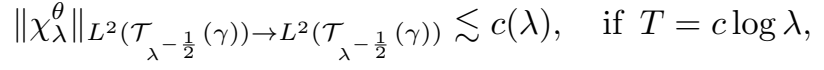

where $c>0$ will be a small constant, chosen, for instance, so that the $\delta>0$ in (3.4) is small. Also, the first part of (3.6) means that we assume that the operators satisfy the bounds when, as in 2.5, we assume that the functions are supported in $\mathcal{T}_{\lambda^{-\frac{1}{2}}}(\gamma)$. Since $\chi(T(\lambda+P))$ has a smooth kernel with $O\left(\lambda^{-N}\right)$ bounds on all derivatives independent of $T \geq 1$, to prove 3.6 , by Euler's formula, it suffices to show that

$$
\left.\left\|\tilde{\chi}_{\lambda}^{\theta}\right\|_{L^{2}\left(\mathcal{T}_{\lambda}-\frac{1}{2}\right.}(\gamma)\right) \rightarrow L^{2}\left(\mathcal{T}_{\lambda-\frac{1}{2}}(\gamma)\right) \lesssim c(\lambda), \quad \text { if } T=c \log \lambda
$$

where

$$
\tilde{\chi}_{\lambda}^{\theta}=\frac{1}{\pi T} \int \widehat{\chi}(t / T) e^{-i \lambda t} Q_{\theta, \lambda} \circ \cos t \sqrt{-\Delta_{g}} d t
$$

We have switched from $\exp \left(i t \sqrt{-\Delta_{g}}\right)$ to $\cos t \sqrt{-\Delta_{g}}$ so that we can use the Hadamard parametrix and the Cartan-Hadamard theorem to lift the calculations that will be needed for $\left.3.6^{\prime}\right)$ up to the universal cover $\left(\mathbb{R}^{n}, \tilde{g}\right)$ of $(M, g)$. This is the approach that was used in [2] and [27.

Let $\{\alpha\}=\Gamma$ denotes the group of deck transformations preserving the associated covering map $\kappa: \mathbb{R}^{n} \rightarrow M$ coming from the exponential map from $\gamma(0)$ associated with the metric $g$ on $M$. The metric $\tilde{g}$ then is its pullback via $\kappa$. Choose also a Dirichlet fundamental domain, $D \simeq M$, for $M$ centered at the lift $\tilde{\gamma}(0)$ of $\gamma(0)$. We shall let $\tilde{\gamma}(t), t \in \mathbb{R}$, denote the lift of the geodesic $\gamma(t), t \in \mathbb{R}$, containing the unit segment $\gamma(t), 0 \leq t \leq 1$ around which our tube is centered. We shall work in geodesic normal coordinates vanishing at $\tilde{\gamma}(0)$ and we may assume that

$$
\tilde{\gamma}(t)=\{(t, 0, \ldots, 0): t \in \mathbb{R}\} .
$$

Let $\Gamma_{\mathcal{T}_{R}(\tilde{\gamma})} \subset \Gamma$ be all those deck transformations for which

$$
\alpha(D) \cap \mathcal{T}_{R}(\tilde{\gamma}) \neq \emptyset \text {, where } \quad R=100 \cdot \operatorname{diam} D .
$$

Here $\mathcal{T}_{R}(\tilde{\gamma})$ denotes the $R$-tube about the geodesic in $\left(\mathbb{R}^{n}, \tilde{g}\right)$, and, since $D$ is a Dirichlet domain

$$
R \approx \operatorname{Inj} M .
$$

We measure distances in $\left(\mathbb{R}^{n}, \tilde{g}\right)$ using its Riemannian distance function $d_{\tilde{g}}(\cdot, \cdot)$. The distance function on $(M, g)$ is similarly denoted by $d_{g}(\cdot, \cdot)$. 
Following [27, we recall also that if $\tilde{x}$ denotes the lift of $x \in M$ to $D$, then we have the following formula

$$
\left(\cos t \sqrt{-\Delta_{g}}\right)(x, y)=\sum_{\alpha \in \Gamma}\left(\cos t \sqrt{-\Delta_{\tilde{g}}}\right)(\tilde{x}, \alpha(\tilde{y}))
$$

Consequently,

$$
\widetilde{\chi}_{\lambda}^{\theta}=\sum_{\alpha \in \Gamma} U_{\alpha}^{\theta, \lambda}
$$

where $U_{\alpha}^{\theta, \lambda}$ is the operator with kernel $U^{\theta, \lambda}(\tilde{x}, \alpha(\tilde{y}))$, where

$$
\begin{aligned}
& U^{\theta, \lambda}(\tilde{x}, \tilde{y})=\frac{1}{\pi T} \int \widehat{\chi}(t / T) e^{i \lambda t}\left(Q_{\theta, \lambda} \circ \cos \left(t \sqrt{-\Delta_{\tilde{g}}}\right)\right)(\tilde{x}, \tilde{y}) d t \\
&=\left(Q_{\theta, \lambda} \circ\left[\frac{1}{\pi T} \int \widehat{\chi}(t / T) e^{i \lambda t}\left(\cos \left(t \sqrt{-\Delta_{\tilde{g}}}\right)\right) d t\right]\right)(\tilde{x}, \tilde{y})
\end{aligned}
$$

If we let $K(\tilde{x}, \tilde{y})$ denote the kernel of the operator in the square brackets, i.e.,

$$
K(\tilde{x}, \tilde{y})=\frac{1}{\pi T} \int \widehat{\chi}(t / T) e^{i \lambda t}\left(\cos \left(t \sqrt{-\Delta_{\tilde{g}}}\right)\right)(\tilde{x}, \tilde{y}) d t
$$

then, as we shall show, one can use the Hadamard parametrix to obtain the uniform bounds

$$
|K(\tilde{x}, \tilde{y})| \leq C T^{-1} \lambda^{\frac{n-1}{2}}\left(d_{\tilde{g}}(\tilde{x}, \tilde{y})\right)^{-\frac{n-1}{2}}, \quad \text { if } \quad d_{\tilde{g}}(\tilde{x}, \tilde{y}) \geq 1
$$

provided that, as we are assuming, $T=c \log \lambda$ with $c>0$ sufficiently small. The estimates also hold when $d_{\tilde{g}}(\tilde{x}, \tilde{y}) \leq 1$, but we shall need this for now.

We claim that these size estimates along with Young's inequality and 2.11) imply that we have the uniform bounds

$$
\left\|U_{\alpha}^{\theta, \lambda}\right\|_{L^{2}\left(\mathcal{T}_{\lambda^{-\frac{1}{2}}}(\gamma)\right) \rightarrow L^{2}\left(\mathcal{T}_{\lambda^{-\frac{1}{2}}}(\gamma)\right)} \leq C T^{-1}\left(1+d_{\tilde{g}}(0, \alpha(0))\right)^{-\frac{n-1}{2}}
$$

when $\alpha \neq$ Identity, and a slightly different argument will be needed to show that the bounds also hold for $\alpha=$ Identity. We shall postpone the simple proof of (3.8) until after we introduce the Hadamard parametrix.

Recall that, by construction, the kernel $Q_{\theta, \lambda}(x, y)$ vanishes if the distance from either $x$

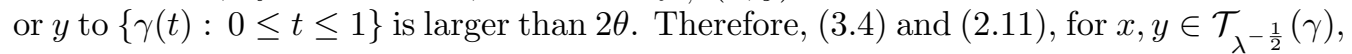
we have

$$
\begin{aligned}
\left|U^{\theta, \lambda}(\tilde{x}, \alpha(\tilde{y}))\right| & \leq C \sup _{x \in \mathcal{T}_{2 \theta}(\gamma), y \in \mathcal{T}_{\lambda^{-} \frac{1}{2}}(\gamma)}|K(\tilde{x}, \alpha(\tilde{y}))| \\
& \leq C T^{-1} \lambda^{\frac{n-1}{2}}\left(d_{\tilde{g}}(0, \alpha(0))\right)^{-\frac{n-1}{2}}
\end{aligned}
$$

due to our assumption (3.4). This and the $L^{1}$ estimates for the kernel of $Q_{\theta, \lambda}$ account for the first inequality here, and the second follows from the fact that $d_{\tilde{g}}(\tilde{x}, \alpha(\tilde{y})) \approx$ $d_{\tilde{g}}(0, \alpha(0))$, if $x, y \in \mathcal{T}_{2 \theta}(\gamma)$, due to our assumptions about $\operatorname{Inj} M$. By using the above estimate and Young's inequality, one obtains 3.8. 
To show it is valid for $\alpha=I$ dentity, choose $\eta \in C_{0}^{\infty}(\mathbb{R})$ satisfying $\eta(s)=1,|s| \leq 2$ and $\eta(s)=0,|s| \geq 3$. Then we can write

$$
K(\tilde{x}, \tilde{y})=\frac{1}{\pi T} \int \eta(t) \widehat{\chi}(t / T) e^{i \lambda t}\left(\cos t \sqrt{-\Delta_{g}}\right)(\tilde{x}, \tilde{y}) d t+R(\tilde{x}, \tilde{y}),
$$

where

$$
R(\tilde{x}, \tilde{y})=\frac{1}{\pi T} \int(1-\eta(t)) \widehat{\chi}(t / T) e^{i \lambda t}\left(\cos t \sqrt{-\Delta_{\tilde{g}}}\right)(\tilde{x}, \tilde{y}) d t
$$

since $\left(\cos t \sqrt{-\Delta_{\tilde{g}}}\right)(\tilde{x}, \tilde{y})=\left(\cos t \sqrt{-\Delta_{g}}\right)(x, y)$ if $d_{g}(x, y) \leq 3$, due to Huygens principle and our assumptions about Inj $M$. The operator with kernel equal to the first term in the formula for $K(\tilde{x}, \tilde{y})$ is obviously bounded from $L^{2}(M)$ to itself with norm $O\left(T^{-1}\right)$ since $\cos t \sqrt{-\Delta_{g}}$ has norm one. Later we shall show, also using the Hadamard parametrix, that

$$
|R(\tilde{x}, \tilde{y})| \leq C T^{-1}, \quad \text { if } \quad d_{g}(x, y) \leq 2,
$$

provided, as we are assuming, $T=c \log \lambda$ with $c>0$ sufficiently small. Using these facts and the fact that $\left\|Q_{\theta, \lambda}\right\|_{L^{2}(M) \rightarrow L^{2}(M)} \leq C$ (by (2.11)), one deduces that $\left(3.8^{\prime}\right)$ also must hold for $\alpha=$ Identity, which completes its proof (apart from showing that (3.8) and $\left(3.8^{\prime}\right)$ are valid as we shall do later).

Since there are only $O(1)$, "translates," $\alpha(D)$, of $D$ that intersect any geodesic ball with arbitrary center of radius $R$ (which was fixed earlier) $1^{1}$ it follows that

$$
\#\left\{\alpha \in \Gamma_{\mathcal{T}_{R}(\tilde{\gamma})}: d_{\tilde{g}}(0, \alpha(0)) \in\left[2^{k}, 2^{k+1}\right]\right\} \leq C 2^{k} .
$$

This is because one can cover the set $\left\{x \in \Gamma_{\mathcal{T}_{R}(\tilde{\gamma})}: d_{\tilde{g}}(0, x) \in\left[2^{k}, 2^{k+1}\right]\right\}$ with $O\left(2^{k}\right)$ balls of radius $R$. Thus, we deduce from $\left(2.1^{\prime}\right)$ and $\left(3.8^{\prime}\right)$ that

$\sum_{\alpha \in \Gamma \mathcal{T}_{R}(\tilde{\gamma})}\left\|U_{\alpha}^{\theta, \lambda}\right\|_{L^{2}\left(\mathcal{T}_{\lambda^{-\frac{1}{2}}}(\gamma)\right) \rightarrow L^{2}\left(\mathcal{T}_{\lambda^{-\frac{1}{2}}}(\gamma)\right)} \lesssim T^{-1} \sum_{1 \leq 2^{k} \leq T} 2^{k} 2^{-k \frac{n-1}{2}} \lesssim c(\lambda), \quad$ if $T=c \log \lambda$,

where the constants $c(\lambda)$ are as in $(1.3)$. Recall that $\left(\cos t \sqrt{-\Delta_{\tilde{g}}}\right)(x, y)=0$ if $d_{\tilde{g}}(x, y)>t$. Here, as in the rest of this section, we are dropping the tildes from our various points $x$, $y$, etc., since all the calculations will be done in $\left(\mathbb{R}^{n}, \tilde{g}\right)$.

Since there are $O\left(\exp \left(c_{0} T\right)\right)$ nonzero $U_{\alpha}^{\theta, \lambda}$ for some fixed $c_{0}$, we deduce that we would finish matters and obtain $\left(3.6^{\prime}\right)$ if we could choose the constant $c$ in definition of $T$ so that for large enough $\lambda$ we have.

$$
\left\|U_{\alpha}^{\theta, \lambda}\right\|_{L^{2}\left(\mathcal{T}_{\lambda^{-\frac{1}{2}}}(\gamma)\right) \rightarrow L^{2}\left(\mathcal{T}_{\lambda^{-\frac{1}{2}}}(\gamma)\right)} \leq \lambda^{-1} \quad \text { if } \alpha \notin \Gamma_{\mathcal{T}_{R}(\tilde{\gamma})} .
$$

We shall need to finally use Toponogov's theorem to do this. We note that, by Proposition 2.1. we have

$$
\left\{r \frac{\xi}{|\xi|}:\left|\frac{\xi}{|\xi|}-(1,0, \ldots, 0)\right| \leq c_{R} \theta_{T},|r| \leq T\right\} \subset \mathcal{T}_{R}(\tilde{\gamma}), \text { if } \theta_{T}=e^{-T}
$$

\footnotetext{
${ }^{1}$ One sees this assertion by noting that, since the $\alpha$ are isometric, the volume, $v$, of $D$ agrees with that of any $\alpha(D), \alpha \in \Gamma$. Similarly, if $d$ denotes the diameter of our Dirichlet domain $D$, then $\alpha(D)$ has the same diameter. Let $B_{\tilde{g}}(P ; R)$ denote the geodesic ball of radius $R$ about some point $P$. Then if $\alpha(D) \cap B_{\tilde{g}}(P ; R) \neq \emptyset$, it follows from the triangle inequality that $\alpha(D) \subset B_{\tilde{g}}(P ; R+d)$. As the $\alpha(D)$ are disjoint, the number of such domains intersecting the ball is therefore bounded from above by $\operatorname{Vol}_{\tilde{g}}\left(B_{\tilde{g}}(P ; R+d)\right) / v$. Since volume comparison theorems (see, e.g. 7]) and our curvature assumptions imply that $\operatorname{Vol}_{\tilde{g}}\left(B_{\tilde{g}}(P ; R+d)\right)$ is bounded by the the volume of balls with this radius in hyperbolic space, $\mathbb{H}^{n}$, the assertion follows.
} 
Recall that the $x$-gradient of the Riemannian distance function, $d_{\tilde{g}}(x, y)$ points in the direction of the tangent vector at $x$ of the geodesic connecting $x$ and $y$. So the last assertion just means that

$$
\min _{ \pm}\left|\frac{\nabla_{x} d_{\tilde{g}}(x, y)}{\left|\nabla_{x} d_{\tilde{g}}(x, y)\right|} \pm \mathbb{1}\right| \geq c_{R} \theta_{T}, \quad \text { if } \quad x=0 \text { and } y \notin \mathcal{T}_{R}(\tilde{\gamma})
$$

with $\mathbb{1}$ denoting the vector $(1,0, \ldots, 0)$. Repeating ourselves, this is just because the geodesic cones of aperture $\approx \theta_{T}$ (measured by the metric $\tilde{g}$ ) with vertex 0 and central directions $\pm 1= \pm \frac{d}{d t} \tilde{\gamma}(t), t=0$, are contained in the intersection of the ball of radius $T$ centered at our origin and $\mathcal{T}_{R}(\tilde{\gamma})$. The same will remain true at any $x$ point on our unit length geodesic $\{\tilde{\gamma}(t): 0 \leq t \leq 1\}$ since we would just be replacing $T$ by a radius in $[T-1, T+1]$. Hence, if we choose $c_{\delta}=c>0$ in the definition of $T=c \log \lambda$ to be small enough so that $c_{R} \theta_{T}=\lambda^{-\delta / 2}$, we have the crucial fact that

$$
\min _{ \pm}\left|\frac{\nabla_{x} d_{\tilde{g}}(x, y)}{\left|\nabla_{x} d_{\tilde{g}}(x, y)\right|} \pm 1\right| \geq \lambda^{-\delta / 2}, \quad \text { if } \quad x=\gamma(t), 0 \leq t \leq 1 \text {, and } y \notin \mathcal{T}_{R}(\tilde{\gamma})
$$

We can take $\delta$ to be any fixed small positive number by adjusting this constant $c$. In particular, we shall want it to be small enough so that both (3.4) and (3.5) are both valid. We should also point out that every time we reduce the size of $c$ this has the effect of improving the Toponogov lower bound 2.7) and so the previous steps of the proof will not be invalidated when we make future reductions.

The pseudo-differential cutoff $Q_{\theta, \lambda}$ occurring in the definition 3.7) of $U_{\alpha}^{\theta, \lambda}$ involves microlocalizing at an angle $\theta=\theta_{\lambda}$ of size $\lambda^{-\delta}$ about our geodesic, which is much smaller than the one occurring in 3.10 if $\lambda$ is large. This will allow us to show that for $\lambda \gg 1$ we have

$$
\left|U^{\theta, \lambda}(x, y)\right| \leq \lambda^{-1} \quad \text { if } \quad d_{\tilde{g}}(x,\{\tilde{\gamma}(t): 0 \leq t \leq 1\}) \leq \lambda^{-\frac{1}{2}}, \text { and } y \notin \mathcal{T}_{R}(\tilde{\gamma}),
$$

which is more than adequate for obtaining 3.9 . As noted before, $U_{\alpha}^{\theta, \lambda}(x, y)$ vanishes identically when $d_{\tilde{g}}(x, y)>T$.

Note that, because of the coordinates we are using, by (2.8), the compound symbol of our pseudo-differential cutoff $Q_{\theta, \lambda}$ satisfies

$$
\begin{aligned}
& Q_{\theta, \lambda}(x, y, \xi)=0 \quad \text { if } d_{\tilde{g}}(x,\{(t, 0, \ldots, 0): 0 \leq t \leq 1\}) \geq C \lambda^{-\delta} \\
& \quad d_{\tilde{g}}(y,\{(t, 0, \ldots, 0): 0 \leq t \leq 1\}) \geq C \lambda^{-\delta}, \text { or } \min _{ \pm}\left| \pm \frac{\xi}{|\xi|}-1\right| \geq C \lambda^{-\delta}
\end{aligned}
$$

for some constant $C$ where $\mathbb{1}=(1,0, \ldots, 0)$ denotes the direction of our unit speed geodesic $\tilde{\gamma}(t)=(t, 0, \ldots, 0)$. Similarly, by 2.10 , since we are taking $\theta=\lambda^{-\delta}$, the symbol also satisfies satisfy the size estimates

$$
\left|D_{x, y}^{\alpha_{1}} D_{\xi}^{\alpha_{2}} Q_{\theta, \lambda}(x, y, \xi)\right| \leq C_{\alpha_{1}, \alpha_{2}} \lambda^{\delta\left(\left|\alpha_{1}\right|+\left|\alpha_{2}\right|\right)}(1+|\xi|)^{-\left|\alpha_{2}\right|} .
$$

At this point we shall follow the argument in Bérard 1] and use the fact that since $\left(\mathbb{R}^{n}, \tilde{g}\right)$ has nonpositive curvature as well as curvature pinched below by -1 we can use the Hadamard parametrix to write the kernel of $\cos t \sqrt{-\Delta_{\tilde{g}}}$ as a Fourier integral whose symbol and phase have derivatives growing at most exponentially in terms of the geodesic distance from the origin. There also will be a remainder with this property, but this will trivial to deal with. 
The phase functions will just involve the Riemannian distance functions $d_{\tilde{g}}(x, y)=r$. In addition to using (3.10), we shall need to use the fact that when derivatives are taken with respect to our coordinate system we have for every multi-index $\alpha$

$$
D_{x}^{\alpha} d_{\tilde{g}}(x, y) \leq C_{\alpha} \exp \left(c_{\alpha} r\right)
$$

for some constants $C_{\alpha}$ and $c_{\alpha}$. This follows from Proposition 3 and Lemma 4 on p. 274 in Bérard [1]. Note that we get from this and (3.10) that, after possibly reducing the size of the constant $c$ in the definition of $T$, we may assume in addition to 3.10 that for large enough $\lambda$ we have

$$
\begin{aligned}
\min _{ \pm}\left|\frac{\nabla_{w} d_{\tilde{g}}(w, y)}{\left|\nabla_{w} d_{\tilde{g}}(w, y)\right|} \pm \mathbb{1}\right| & \geq \lambda^{-\delta / 2}, \\
& \text { if } d_{\tilde{g}}(w,\{\tilde{\gamma}(t), 0 \leq t \leq 1\})=O\left(\lambda^{-\frac{1}{2}}\right), \text { and } y \notin \mathcal{T}_{R}(\tilde{\gamma}),
\end{aligned}
$$

since we are assuming that $\delta$ is smaller than $1 / 2$.

Next, following Bérard ${ }^{2}[1$, we can use the Hadamard parametrix to write for, say, $|t|$ or $d_{\tilde{g}}(x, y)$ larger than 1 ,

$$
\left(\cos t \sqrt{-\Delta_{\tilde{g}}}\right)(x, y)=\sum_{ \pm} \int_{\mathbb{R}} e^{i \tau\left(d_{\tilde{g}}(x, y) \pm t\right)} a_{ \pm}(x, y, \tau) d \tau+R(t, x, y),
$$

where, if $|t|<T$ as well and if $N_{0} \in \mathbb{N}$ is fixed

$$
\begin{aligned}
& \left|\left(\frac{\partial}{\partial \tau}\right)^{j} a_{ \pm}(x, y, \tau)\right| \leq C_{j}\left(1+d_{\tilde{g}}(x, y)\right)^{-\frac{n-1}{2}}(1+|\tau|)^{\frac{n-1}{2}-j}, \quad \text { and } \\
& \quad\left|D_{x}^{\alpha_{1}}\left(\frac{\partial}{\partial \tau}\right)^{j} a_{ \pm}(x, y, \tau)\right| \leq C_{j, \alpha_{1}} \exp \left(c_{N_{0}} T\right)(1+|\tau|)^{\frac{n-1}{2}-j}, \quad 0<\left|\alpha_{1}\right| \leq N_{0},
\end{aligned}
$$

and we may assume that the remainder satisfies

$$
\left|\left(\frac{\partial}{\partial t}\right)^{j} R(t, x, y)\right| \leq C_{N_{0}} \exp \left(c_{N_{0}} T\right), \quad \text { if } j \leq N_{0} .
$$

The various constants here are independent of $T$, but the exponential rate of growth in this parameter, may depend on the number of space-time derivatives taken. These bounds were established in [1].

Fix now $\varrho \in C^{\infty}(\mathbb{R})$ satisfying $\varrho=1$ on $[-R / 2, R / 2]$ and supp $\varrho \subset[-2 R, 2 R]$. Then, by Huygens' principle and (3.7)

$$
\begin{array}{r}
U^{\theta, \lambda}(x, y)=\frac{1}{\pi T} \int(1-\varrho(t)) \widehat{\chi}(t / T) e^{i \lambda t}\left(Q_{\theta, \lambda} \circ \cos \left(t \sqrt{-\Delta_{\tilde{g}}}\right)\right)(x, y) d t \\
\text { if } x \in \mathcal{T}_{\lambda^{-\frac{1}{2}}}(\gamma), \text { and } y \notin \mathcal{T}_{R}(\tilde{\gamma}) .
\end{array}
$$

If we let $R_{t}$ denote the integral operator with kernel $R(t, x, y)$, then, by 2.11 and (3.16), we have

$$
\left|\partial_{t}^{j}\left(Q_{\theta, \lambda} \circ R_{t}\right)(x, y)\right| \leq C \exp (c T), \quad j=0,1,2
$$

\footnotetext{
${ }^{2}$ We are using the phase functions $\theta\left(d_{\tilde{g}} \pm t\right)$ for our Fourier integrals for the sake of convenience, instead of $\theta\left(d_{\tilde{g}}^{2}-t^{2}\right)$ as in [1], but our formulation follows from Bérard's and an obvious change of variables.
} 
By a simple integration by parts argument in $t$, we see from this that if we replace $\cos t \sqrt{-\Delta_{\tilde{g}}}$ by $R_{t}$ in $\left(3.7^{\prime}\right)$, we would obtain a kernel $R(x, y)$ satisfying

$$
|R(x, y)| \leq B \exp (B T) \lambda^{-2}
$$

for some constant $B$.

Since this can be made small compared to $\lambda^{-1}$ for large $\lambda$ if the constant $c$ in the definition of $T$ is chosen to be small enough, we would obtain $\left(3.9^{\prime}\right)$ if we could obtain similar bounds when we replace $\cos t \sqrt{-\Delta_{\tilde{g}}}$ by each of the Fourier integral operators whose kernels are the two main terms in 3.14 coming from the sum over \pm .

To simplify the calculation that will be involved let us make a couple of trivial reductions.

The first involves the pseudo-differential cutoff. We recall that the kernel of $Q_{\theta, \lambda}$ is given by

$$
Q_{\theta, \lambda}(x, w)=\int e^{i\langle x-w, \xi\rangle} Q_{\theta, \lambda}(x, w, \xi) d \xi
$$

where the symbol satisfies (3.11) and (3.12). Since we are assuming that $\delta$ is smaller than $1 / 2$ it follows that if

$$
\widetilde{Q}_{\theta, \lambda}(x, w)=\varrho\left(\lambda^{\frac{1}{2}} d_{\tilde{g}}(x, w)\right) \int e^{i\langle x-w, \xi\rangle} Q_{\theta, \lambda}(x, w, \xi) d \xi
$$

then $R_{\theta}(x, w)=Q_{\theta, \lambda}(x, w)-\widetilde{Q}_{\theta, \lambda}(x, w)$ is also supported near our unit geodesic and satisfies

$$
\left|\partial_{x, w}^{\alpha} R_{\theta}(x, w)\right| \leq C_{N, \alpha} \lambda^{-N} \quad \forall \alpha, N
$$

by (2.11). Therefore, by an argument similar to the one just given, if we replace $Q_{\theta, \lambda}$ by $\widetilde{Q}_{\theta, \lambda}$ and $\cos t \sqrt{-\Delta_{\tilde{g}}}$ in $\left(3.7^{\prime}\right)$ by the sum of the two Fourier integrals in (3.14), then the difference between the kernel in $\left(3.7^{\prime}\right)$ and the resulting kernel will satisfy the bounds in (3.17). Thus, in what follows, we may replace $Q_{\theta, \lambda}$ by $\widetilde{Q}_{\theta, \lambda}$ and ignore the remainder term in (3.14) in our calculations. We have made this reduction to make it simpler to apply $3.10^{\prime}$.

For the Fourier integral operators we shall note that we can make a Littlewood-Paley decomposition. Specifically, if $\beta \in C_{0}^{\infty}(\mathbb{R})$, satisfies

$$
\beta(s)=1 \text {, if } s \in[1 / 2,2], \quad \text { and } \beta(s)=0, \text { if } s \notin[1 / 4,4],
$$

then we note that

$$
T^{-1}\left|\int(1-\varrho(t)) \hat{\chi}(t / T)(1-\beta(|\tau| / \lambda)) e^{i t(\lambda \pm \tau)} d t\right| \leq C_{N}(\lambda+|\tau|)^{-N}, \quad \forall N,
$$

where the $C_{N}$ are independent of $T \geq 1$. As a result, we may also multiply the symbols in the Fourier integrals by $\beta(|\tau| / \lambda)$ in our calculation at the expense of introducing another error satisfying the bounds which are better than those in (3.17).

Summarizing, in proving the remaining estimate to establish $\left(3.9^{\prime}\right)$, we may replace $Q_{\theta, \lambda}$ by $\widetilde{Q}_{\theta, \lambda}$ and the two Fourier integrals in (3.14) by the ones where the symbol is multiplied by $\beta(|\tau| / \lambda)$. If we also abuse notation a bit and multiply the symbol of $Q_{\theta, \lambda}$ 
by a smooth factor, but still write it as $Q_{\theta, \lambda}$, to take into account that the integrations are given with respect to the volume element, we have reduced matters to showing that

$$
\begin{aligned}
& T^{-1}\left|\iiint(1-\varrho(t)) \hat{\chi}(t / T) e^{i t(\lambda \pm \tau)} e^{i x \cdot \xi} b_{ \pm}(\lambda, x, w, y, \tau, \xi) e^{i \phi(w, y, \tau, \xi)} d w d \tau d \xi d t\right| \\
& \leq B \exp (B T) \lambda^{-2}, \quad \text { if } d_{\tilde{g}}(x,\{\tilde{\gamma}(t): 0 \leq t \leq 1\}) \leq \lambda^{-\frac{1}{2}} \text { and } y \notin \mathcal{T}_{R}(\tilde{\gamma}),
\end{aligned}
$$

where the symbol here is given by

$$
b_{ \pm}(\lambda, x, w, y, \tau, \xi)=\varrho\left(\lambda^{\frac{1}{2}} d_{\tilde{g}}(x, w)\right) \widetilde{Q}_{\theta, \lambda}(x, w, \xi) a_{ \pm}(w, y, \tau) \beta(|\tau| / \lambda),
$$

and the phase here is given by

$$
\phi(w, y, \tau, \xi)=\tau d_{\tilde{g}}(w, y)-w \cdot \xi .
$$

To proceed, we note that, due to the $\varrho\left(\lambda^{\frac{1}{2}} \cdot\right)$ cutoff, the first condition in $\left(\sqrt{3.10^{\prime}}\right)$ is fulfilled on the support of the integrand and we are assuming the other one in (3.17). Therefore, if we recall the $\xi$-support assumptions in (3.11) for $Q_{\theta, \lambda}$, we can use $\left(3.10^{\prime}\right)$ to see that, on the support of the integrand, there must be a positive constant $c_{0}$ so that for large $\lambda$

$$
\begin{aligned}
& \left|\nabla_{w} \phi(w, y, \tau, \xi)\right| \geq c_{0} \lambda^{-\frac{\delta}{2}}(|\lambda|+|\tau|+|\xi|), \quad \text { if } b_{ \pm} \neq 0, \\
& \quad d_{\tilde{g}}(x,\{\tilde{\gamma}(t): 0 \leq t \leq 1\}) \leq \lambda^{-\frac{1}{2}}, \text { and } y \notin \mathcal{T}_{R}(\tilde{\gamma}) .
\end{aligned}
$$

Since $Q_{\theta, \lambda}, d_{\tilde{g}}$ and $a_{ \pm}$satisfy the size estimates in (3.12), (3.11) and (3.15), respectively, we can integrate by parts a finite number of times to obtain (3.19). We choose $N_{0}$ in (3.15) to be large enough to handle the number of integration by parts which depends only on the dimension.

To wrap up matters, apart from proving the two Propositions, to prove the KakeyaNikodym estimates we still have to prove (3.8) and $\left(3.8^{\prime \prime}\right)$. We shall use a variation on the argument that we just gave which is considerably easier.

To prove the former we use 3.14 . If we replace $\left(\cos t \sqrt{-\Delta_{\tilde{g}}}\right)(x, y)$ in the definition of $K(x, y)$ by the remainder term in (3.14), the resulting expression will clearly be $O\left(\exp \left(c_{0} T\right)\right)$, which clearly is smaller than the right side of $(3.8)$ if $T=c \log \lambda$ with $c>0$ small provided that $d_{\tilde{g}}(x, y) \leq T$, as we may assume since $K(x, y)$ vanishes otherwise by Huygens' principle. If we replace $\left(\cos t \sqrt{-\Delta_{\tilde{g}}}\right)(x, y)$ by the main term in the Hadamard parametrix, (3.14), the resulting expression equals half of the sum over \pm of

$$
\int_{-\infty}^{\infty} e^{i \tau d_{\tilde{g}}(x, y)} \chi(T(\lambda \pm \tau)) a_{ \pm}(x, y, \tau) d \tau,
$$

and, using the first part of 3.15 with $j=0$, this is also seen to satisfy the bounds in (3.8) since $\chi \in \mathcal{S}(\mathbb{R})$.

To prove $\left(3.8^{\prime \prime}\right)$, one uses a similar argument. Using $\left(3.16^{\prime}\right)$ for $j=0,1$ one sees by an integration by parts argument in $t$ that the contribution of the remainder term to $\left(3.8^{\prime \prime}\right)$ is $O\left(\lambda^{-1} \exp (c T)\right)$, which is much better than the bounds in (3.8 $)$ if $T=c \log \lambda$ with $c$ small. Since we are assuming that $\left|d_{\tilde{g}} \pm t\right| \geq 1$ on the support of the integral defining $R(x, y)$, if we plug the main term of the Hadamard parametrix (3.14) and integrate by parts in $\tau$, we see that its contribution is $O\left(\lambda^{-N}\right)$ for any $N$ since the Fourier transform of $t \rightarrow T^{-1}(1-\eta(t)) \hat{\chi}(t / T)$ is bounded and rapidly decreasing at infinity. Thus, its 
contribution also is much better than the bounds posited in $\left(3.8^{\prime \prime}\right)$, which finishes its proof.

Let us conclude this section by showing how these arguments imply the two-dimensional restriction estimates 1.4 . If $\rho \in \mathcal{S}(\mathbb{R})$ is as in 2.1 , the bounds for eigenfunctions would follow from showing that we have the uniform bounds

$$
\|\rho(T(\lambda-P)) f\|_{L^{2}(\gamma)} \leq C(\lambda / \log \lambda)^{\frac{1}{4}}\|f\|_{L^{2}(M)}, \text { if } \gamma \in \Pi, \text { and } T \approx \log \lambda .
$$

Since $\rho(0)=1$ this implies

$$
\left\|E_{\left[\lambda, \lambda+(\log \lambda)^{-1}\right]} f\right\|_{L^{2}(\gamma)} \lesssim(\lambda / \log \lambda)^{\frac{1}{4}}\|f\|_{L^{2}(M)},
$$

which in turn, by a routine argument (cf. 30]) implies that quasi-modes satisfying the condition 1.5 also satisfy the bounds in 1.4.

If one uses the argument from the first part of the proof of $(1.3$ ) along with 2.13 , one finds that

$$
\left\|\left(I-Q_{\theta, \lambda}\right) \circ \rho(T(\lambda-P)) f\right\|_{L^{2}(\gamma)} \lesssim \varepsilon \theta^{-\frac{1}{2}-\varepsilon}\|f\|_{L^{2}(M)},
$$

which is better than the bounds in 3.20 assuming, as we shall, that $\theta=\lambda^{-\frac{1}{2}+\delta_{0}}$ for some $\delta_{0}>0$ and $\varepsilon<\delta_{0}$. Thus, we have reduced the proof of 3.20 to showing that for suitable $\theta$ we have

$$
\left\|Q_{\theta, \lambda} \circ \rho(T(\lambda-P)) f\right\|_{L^{2}(\gamma)} \leq C(\lambda / \log \lambda)^{\frac{1}{4}}\|f\|_{L^{2}(M)}
$$

assuming that, as before, $T=c \log \lambda$, for some fixed $c>0$.

By a routine $T T^{*}$ argument if $\chi=|\rho|^{2}$, this is equivalent to showing that

$$
\begin{aligned}
\left(\int_{0}^{1}\left|\int_{0}^{1}\left(Q_{\theta, \lambda} \circ \chi(T(\lambda-P)) \circ Q_{\theta, \lambda}^{*}\right)\left(\gamma(s), \gamma\left(s^{\prime}\right)\right) h\left(s^{\prime}\right) d s^{\prime}\right|^{2} d s\right)^{\frac{1}{2}} & \\
& \leq C(\lambda / \log \lambda)^{\frac{1}{2}}\|h\|_{L^{2}([0,1])} .
\end{aligned}
$$

Since $\chi(T(\lambda+P))(x, y)=O\left(\lambda^{-N}\right)$, arguing as before, we see that, modulo a trivial error, we can express the kernel here as $\sum_{\alpha \in \Gamma} U_{\alpha}^{\theta, \lambda}$ where now

$$
U_{\alpha}^{\theta, \lambda}\left(s, s^{\prime}\right)=\left(Q_{\theta, \lambda} \circ \chi(T(\lambda-P))(\cdot, \alpha(\cdot)) \circ Q_{\theta, \lambda}^{*}\right)\left(\gamma(s), \gamma\left(s^{\prime}\right)\right) .
$$

If $\alpha \neq$ Identity, the earlier arguments yield the uniform bounds

$$
\left|U_{\alpha}^{\theta, \lambda}\left(s, s^{\prime}\right)\right| \leq C T^{-1} \lambda^{\frac{1}{2}}\left(d_{\tilde{g}}(0, \alpha(0))\right)^{-\frac{1}{2}}, \quad 0 \leq s, s^{\prime} \leq 1,
$$

and so

$$
\begin{aligned}
&\left(\int_{0}^{1} \mid \int_{0}^{1} U_{\alpha}^{\theta, \lambda}\left(s, s^{\prime}\right)\right.\left.\left.h\left(s^{\prime}\right) d s^{\prime}\right|^{2} d s\right)^{\frac{1}{2}} \\
& \leq C T^{-1} \lambda^{\frac{1}{2}}\left(1+d_{\tilde{g}}(0, \alpha(0))^{-\frac{1}{2}}\|h\|_{L^{2}([0,1])}, \quad \alpha \neq \text { Identity } .\right.
\end{aligned}
$$

We claim that the same bounds hold for $\alpha=$ Identity. Indeed if, as before, $\eta \in C_{0}^{\infty}(\mathbb{R})$ satisfies $\eta(s)=1,|s| \leq 2$ and $\eta(s)=0,|s| \leq 3$, it follows that when $\alpha$ is the identity the 
kernel can be split as

$$
\begin{array}{r}
U_{I d}^{\theta, \lambda}\left(s, s^{\prime}\right)=\frac{1}{\pi T}\left(Q_{\theta, \lambda} \circ\left(\int \eta(t) \widehat{\chi}(t / T) \cos t \sqrt{-\Delta_{g}} d t\right) \circ Q_{\theta, \lambda}^{*}\right)\left(\gamma(s), \gamma\left(s^{\prime}\right)\right) \\
+R\left(s, s^{\prime}\right)
\end{array}
$$

where, by the proof of $3.8^{\prime \prime}$

$$
\left|R\left(s, s^{\prime}\right)\right| \leq C T^{-1}, \quad \text { if } \quad 0 \leq s, s^{\prime} \leq 1 .
$$

Since Inj $M \geq 10$ one can use a parametrix for $\cos t \sqrt{-\Delta_{g}}$ and stationary phase and argue like in the proof of [20, Lemma 5.1.3] to see that the first term in the right side of $\sqrt{3.23}$ is $O\left(\lambda^{\frac{1}{2}}\left(d_{g}\left(\gamma(s), \gamma\left(s^{\prime}\right)\right)\right)^{-\frac{1}{2}}\right)=O\left(\lambda^{\frac{1}{2}}\left|s-s^{\prime}\right|^{-\frac{1}{2}}\right)$, and so, by Young's inequality, 3.22 is valid for $\alpha=$ Identity as well.

If $U_{\alpha}^{\theta, \lambda}$ are the operators with these kernels our earlier arguments give, analogous to what happened before,

$$
\sum_{\alpha \in \Gamma_{\mathcal{T}_{R}(\tilde{\gamma})}}\left\|U_{\alpha}^{\theta, \lambda}\right\|_{L^{2}(\gamma) \rightarrow L^{2}(\gamma)} \lesssim T^{-1} \lambda^{\frac{1}{2}} \sum_{1 \leq 2^{k} \leq T} 2^{k} 2^{-\frac{k}{2}} \lesssim(\lambda / \log \lambda)^{\frac{1}{2}}, \quad \text { if } T \approx \log \lambda .
$$

By the support properties of $Q_{\theta, \lambda}(x, y), 2.11$ and $\left(3.9^{\prime}\right)$, all the other terms are $O\left(\lambda^{-1}\right)$ if the various parameters are chosen as before. Hence, if, as before, $T=c \log \lambda$ with $c>0$ sufficiently small, we have

$$
\sum_{\alpha \notin \Gamma_{\mathcal{T}_{R}(\tilde{\gamma})}}\left\|U_{\alpha}^{\theta, \lambda}\right\|_{L^{2}(\gamma) \rightarrow L^{2}(\gamma)} \leq 1
$$

which along with the previous bound (and the fact that $\left.\chi(T(\lambda+P))\left(\gamma(s), \gamma\left(s^{\prime}\right)\right)=O(1)\right)$ gives us 3.21), which completes the proof of the restriction estimates.

This completes the proof of Theorem 1.1, apart from the two Propositions, which we shall handle next.

\section{Proof of Proposition 2.1.}

Recall that we are trying to show that

$$
C\left(\theta_{T} ; T\right) \subset \mathcal{T}_{R}(\tilde{\gamma}), \quad \text { if } \sin \frac{1}{2} \theta_{T}=\frac{\sinh \frac{1}{2} R}{\sinh T} .
$$

Where we can take $P$, the vertex of $C\left(\theta_{T} ; T\right)$, to be $\tilde{\gamma}(0)$, and $\tilde{\gamma}(t), t \in \mathbb{R}$, to be the geodesic we have just been working with. $C(\theta ; T)$ then is the intersection of the geodesic ball of radius $T>0$ about our origin with the cone of aperture $\theta$ about $\tilde{\gamma}$, which has coordinates $\{(t, 0, \ldots, 0): t \in \mathbb{R}\}$ in the geodesic normal coordinate system we are using. Also, $\mathcal{T}_{R}(\tilde{\gamma})$ denotes the closed tube of fixed radius $R>0$ about $\tilde{\gamma}$.

Since $T \rightarrow \theta_{T}$ is monotonically decreasing, it suffices to show that a point $Q$ with coordinates $T \omega, \omega \in S^{n-1}$, belongs to $\mathcal{T}_{R}(\tilde{\gamma})$ if the angle, $\varangle(\omega, \mathbb{1})$, is $\leq \theta_{T}$. In other words, to obtain (2.7), it suffices to show that

$$
\Sigma\left(T ; \theta_{T}\right) \subset \mathcal{T}_{R}(\tilde{\gamma})
$$

if $\Sigma(T, \theta)$ denotes all points $Q$ with coordinates $T \omega$ satisfying $\varangle(\omega, \mathbb{1}) \leq \theta$. 
Clearly $\Sigma(T ; \theta) \subset \mathcal{T}_{R}(\tilde{\gamma})$ when $\theta$ is very small (depending on $T$ ). So choose the maximal $\Theta_{T} \leq \pi / 2$ so that $\Sigma(T ; \theta) \subset \mathcal{T}_{R}(\tilde{\gamma})$ when $0<\theta<\Theta_{T}$. It follows that there must be a point $Q$ with coordinates, $T \omega_{0}$, satisfying $\varangle\left(\omega_{0}, 1\right)=\Theta_{T}$ and $d_{\tilde{g}}(Q, \tilde{\gamma})=R$. Also, (4.1) is valid when $\theta_{T}$ is replaced by $\Theta_{T}$ and so we would have (5.14) and be done if we could show that

$$
\Theta_{T} \geq \theta_{T}
$$

At this point, we shall use Toponogov's theorem. First consider the geodesic triangle, $\triangle_{T, \Theta_{T}}^{\tilde{g}}$, in $\left(\mathbb{R}^{n}, \tilde{g}\right)$ with vertices $Q$ and the point with coordinates 0 and the $P$ point with coordinates $(T, 0, \ldots, 0)$. It is an isosceles triangle since the geodesics connecting the point with coordinates 0 with $P$ and $Q$, respectively, each have length $T$. The point $P$ lies on $\tilde{\gamma}$ and hence if $\tilde{\gamma}_{o p p}$ is the third side of our geodesic triangle, which connects $P$ and $Q$, we must have that its length, $\ell\left(\tilde{\gamma}_{o p p}\right)$ satisfies

$$
\ell\left(\tilde{\gamma}_{o p p}\right)=d_{\tilde{g}}(P, Q) \geq R
$$

since, as we pointed out before, we must have $d_{\tilde{g}}(Q, \tilde{\gamma})=R$. The angle at the vertex whose coordinates are the origin, by construction, is $\Theta_{T}$, and the two sides passing through it each have length $T$. The third side of our isosceles triangle, $\tilde{\gamma}_{o p p}$, is called a "Rauch hinge." See Figure 1 .
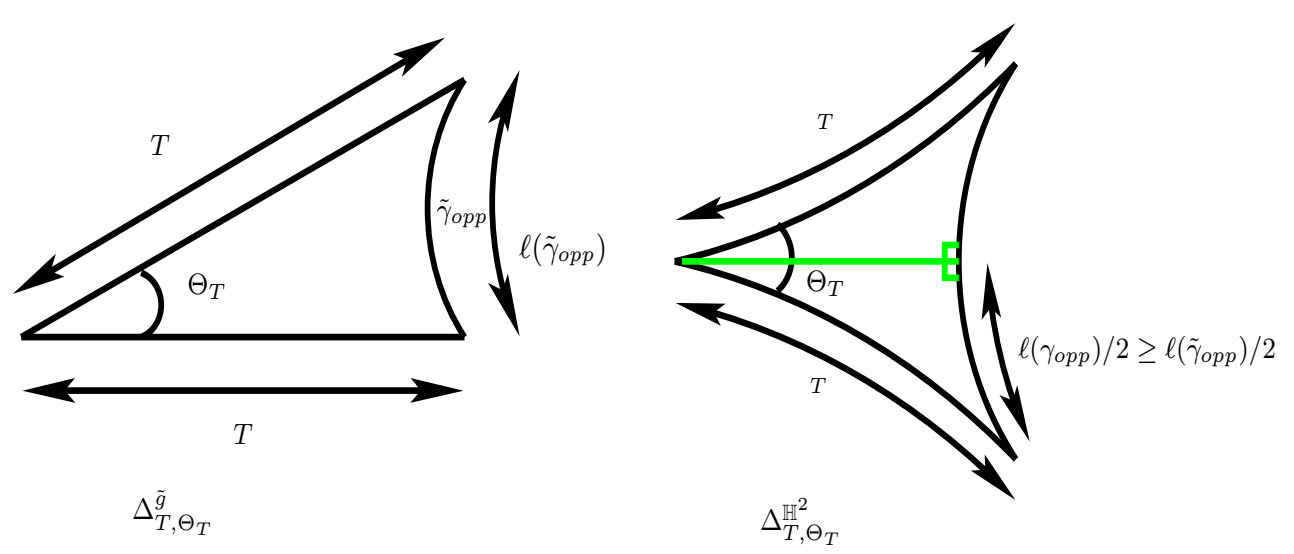

Figure 1. Rauch hinges

Consider as well, an isosceles triangle, $\triangle_{T, \Theta_{T}}^{\mathbb{H}^{2}}$, in two-dimensional hyperbolic space, $\mathbb{H}^{2}$, having two sides of equal length $T$, angle $\Theta_{T}$ at the associated vertex and "Rauch hinge" $\gamma_{o p p}$, with length $\ell\left(\gamma_{o p p}\right)$. By Toponogov's theorem (see [8, Theorem $\left.2.2(\mathrm{~B})\right]$ ), since we are assuming that the sectional curvatures of $\left(\mathbb{R}^{n}, \tilde{g}\right)$ satisfy $-1 \leq K \leq 0$, we must have

$$
\ell\left(\gamma_{\text {opp }}\right) \geq \ell\left(\tilde{\gamma}_{\text {opp }}\right) \geq R
$$

By properties of isosceles triangles in $\mathbb{H}^{2}$, the ray bisecting the triangle at the vertex spanned by the two sides of equal length $T$ must intersect the Rauch hinge, $\gamma_{o p p} \in$ $\triangle_{T, \Theta_{T}}^{\mathbb{H}^{2}}$, orthogonally at its midpoint (see Figure 1 . Consequently, by the law of sines for 
hyperbolic space, we must have

$$
\sin \frac{1}{2} \Theta_{T}=\frac{\sinh \left(\ell\left(\gamma_{\text {opp }}\right) / 2\right)}{\sinh T} \geq \frac{\sinh \frac{1}{2} R}{\sinh T}=\sin \frac{1}{2} \theta_{T} .
$$

Thus, 4.2 is valid and the proof of Proposition 2.1 is complete.

\section{Proof of Proposition 2.2.}

As a first step in the proof of Proposition 2.2, let us make a preliminary reduction. If $\beta$ is a Littlewood-Paley bump function as in (3.18), we claim that it suffices to prove the following dyadic versions of the two inequalities in the Proposition:

$$
\left\|\int a(t) e^{-i t \lambda}\left(I-Q_{\theta, \lambda}\right) \circ \beta(P / \lambda) e^{i t P} f d t\right\|_{L^{2}\left(\mathcal{T}_{\lambda^{-\frac{1}{2}}}(\gamma)\right)} \leq C_{\delta_{0}} \lambda^{-\frac{1}{4}} \theta^{-\frac{1}{2}}\|f\|_{L^{2}(M)},
$$

and

$$
\left\|\int a(t) e^{-i t \lambda}\left(I-Q_{\theta, \lambda}\right) \circ \beta(P / \lambda) e^{i t P} f d t\right\|_{L^{2}(\gamma)} \leq C_{\delta_{0}, \varepsilon} \theta^{-\frac{1}{2}-\varepsilon}\|f\|_{L^{2}(M)},
$$

assuming, as in the Proposition that $\theta \geq \lambda^{-\frac{1}{2}+\delta_{0}}$ with $\delta_{0}, \varepsilon>0$, and that $c_{0}>0$ in 2.9 is sufficiently small.

To verify this claim, we note that

$$
\int a(t) e^{-i \lambda t}(1-\beta)(P / \lambda) e^{i t P} d t
$$

has kernel

$$
\sum \widehat{a}\left(\lambda-\lambda_{j}\right)(1-\beta)\left(\lambda_{j} / \lambda\right) e_{j}(x) \overline{e_{j}(y)} .
$$

Since $a \in C_{0}^{\infty}(\mathbb{R})$ and $\beta$ is as in 3.18 ,

$$
\left|\widehat{a}\left(\lambda-\lambda_{j}\right)(1-\beta)\left(\lambda_{j} / \lambda\right)\right| \leq C\left(1+\lambda+\lambda_{j}\right)^{-n-2} .
$$

Since, by the Weyl formula, we have

$$
\sum_{\lambda_{j} \in[\lambda, \lambda+1]}\left|e_{j}(x) e_{j}(y)\right| \leq C(1+\lambda)^{n-1},
$$

we conclude that the kernel of the operator given by $(5.3)$ is $O\left(\lambda^{-1}\right)$. By the first part of (2.11), the same is true for the kernel of $Q_{\theta, \lambda}$ composed on the left with this operator, and therefore

$$
\int a(t) e^{-i \lambda t}\left(I-Q_{\theta, \lambda}\right) \circ(1-\beta)(P / \lambda) e^{i t P} d t
$$

must have a $O\left(\lambda^{-1}\right)$ kernel. This means that it enjoys better bounds than those posited in Proposition 2.2, which gives us our claim that we just need to prove (5.1) and (5.2).

These two inequalities will be a simple consequence of the following

Lemma 5.1. Fix $0<\delta_{0}<1 / 2$, and assume that $\theta \geq \lambda^{-\frac{1}{2}+\delta_{0}}$ and that $\beta$ is as in (3.18). Then we have the following estimates which are uniform in $\gamma \in \Pi$. First, there is a 
uniform $C_{0}<\infty$ so that for large $\lambda$ we have

$$
\begin{aligned}
\left|\left(\left(I-Q_{\theta, \lambda}\right) \circ \beta(P / \lambda) e^{i t P} \circ\left(I-Q_{\theta, \lambda}\right)^{*}\right)(x, y)\right| & \leq C_{N, \delta_{0}} \lambda^{-N} \quad \forall N, \\
& \text { if } C_{0} \lambda^{-\frac{1}{2}} \theta^{-1} \leq|t| \leq 1, \text { and } x, y \in \mathcal{T}_{\lambda^{-\frac{1}{2}}}(\gamma) .
\end{aligned}
$$

Also, fix $0<\varepsilon<\delta_{0}$. Then for $s, s^{\prime} \in[0,1]$ and large $\lambda$ we have

$$
\begin{array}{r}
\left|\left(\left(I-Q_{\theta, \lambda}\right) \circ \beta(P / \lambda) e^{i t P} \circ\left(I-Q_{\theta, \lambda}\right)^{*}\right)\left(\gamma(s), \gamma\left(s^{\prime}\right)\right)\right| \leq C_{N, \delta_{0}} \lambda^{-N} \quad \forall N, \\
\text { if } \lambda^{-1} \theta^{-2-\varepsilon} \leq\left|s-s^{\prime}\right| \leq 1 .
\end{array}
$$

Remark. There is a simple explanation for the lower bounds for the two time scales in this lemma. The one in (5.4) is a lower bound for the escape time for balls of radius $(\theta \lambda)^{-1}$ to exit $\mathcal{T}_{\lambda^{-\frac{1}{2}}}(\gamma)$ if they are traveling along geodesics forming an angle $\geq \theta$ from its center, $\gamma$. Apart from the the $\varepsilon>0$, the lower bound for $\left|s-s^{\prime}\right|$ in (5.5) is a lower bound for them to escape from $\gamma$. See Figure 2. These escape times are much smaller than 1 due to our assumption that $\theta \geq \lambda^{-\frac{1}{2}+\delta_{0}}$. Also, as $\theta$ increases the ball radius and the escape route both become more favorable.

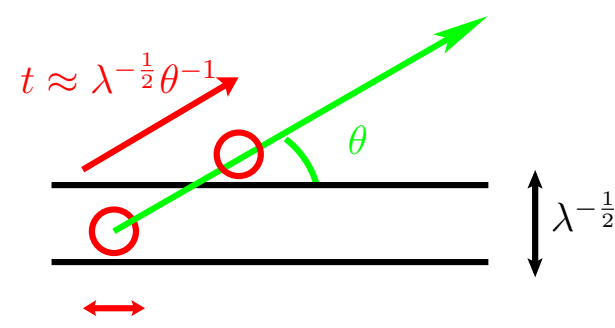

$(\lambda \theta)^{-1}$

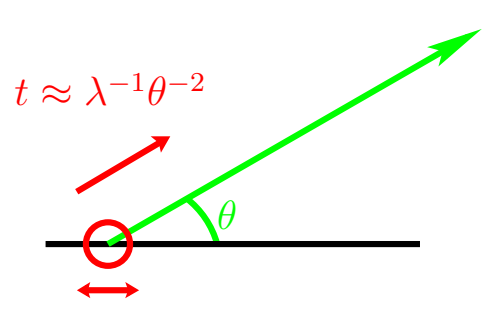

$(\lambda \theta)^{-1}$

Figure 2. Escape times

Proof of 5.1 and 5.2. By using smooth cutoffs in the $t$ variable and the fact that the half-wave operators $e^{i t P}$ are bounded on $L^{2}(M)$ with norm 1 , we deduce that in proving (5.1) and (5.2), we may assume that the smooth function $a$ there is supported in $(-1 / 10,1 / 10)$. The first inequality, (5.1), then is just the statement that

$$
\left\|\left(I-Q_{\theta, \lambda}\right) \widehat{a}(P-\lambda) \beta(P / \lambda)\right\|_{L^{2}(M) \rightarrow L^{2}\left(\mathcal{T}_{\lambda}-\frac{1}{2}(\gamma)\right)}=O\left(\lambda^{-\frac{1}{4}} \theta^{-\frac{1}{2}}\right) .
$$

If $\widetilde{a}=a(\cdot) * \overline{a(-\cdot)}$ and $\widetilde{\beta}=|\beta|^{2}$, then a routine $T T^{*}$ argument shows that this is equivalent to

$$
\begin{aligned}
\|\left(I-Q_{\theta, \lambda}\right) & \circ|\widehat{a}|^{2}(P-\lambda) \widetilde{\beta}(P / \lambda) \circ\left(I-Q_{\theta, \lambda}\right)^{*} f \|_{L^{2}\left(\mathcal{T}_{\lambda^{-\frac{1}{2}}}(\gamma)\right)} \\
& =\left\|\int \widetilde{a}(t) e^{-i \lambda t}\left(I-Q_{\theta, \lambda}\right) \circ \widetilde{\beta}(P / \lambda) e^{i t P} \circ\left(I-Q_{\theta, \lambda}\right)^{*} f d t\right\|_{L^{2}\left(\mathcal{T}_{\lambda^{-\frac{1}{2}}}(\gamma)\right)} \\
& \leq C \lambda^{-\frac{1}{2}} \theta^{-1}\|f\|_{L^{2}}, \quad \text { if } \operatorname{supp} f \subset \mathcal{T}_{\lambda^{-\frac{1}{2}}}(\gamma) .
\end{aligned}
$$


The function $\widetilde{a}$ is supported in $|t| \leq 1$ and $\widetilde{\beta}$ is as in $(3.18)$ if $\beta$ satisfies those conditions. Therefore, we can use 5.4 to see that for $f$ supported in $\mathcal{T}_{\lambda^{-\frac{1}{2}}}(\gamma)$ we have that the left side of this inequality is dominated by

$$
\begin{aligned}
\int_{|t| \leq C_{0} \lambda^{-\frac{1}{2}} \theta^{-1}}\left\|\left(I-Q_{\theta, \lambda}\right) \circ \widetilde{\beta}(P / \lambda) e^{i t P} \circ\left(I-Q_{\theta, \lambda}\right)^{*} f\right\|_{L^{2}(M)} d t+ & O\left(\lambda^{-N}\right)\|f\|_{L^{2}(M)} \\
& \lesssim \lambda^{-\frac{1}{2}} \theta^{-1}\|f\|_{2},
\end{aligned}
$$

using in the last step the fact that $e^{i t P}$ is unitary and the fact that the operators $\left(I-Q_{\theta, \lambda}\right)$ and $\left(I-Q_{\theta, \lambda}\right)^{*}$ are uniformly bounded on $L^{2}$ for $\theta$ as above by virtue of (2.11). Hence, we have 5.6 .

To prove (5.2), we use another $T T^{*}$ argument to see that the inequality is equivalent to the statement that for $n=2$ we have

$$
\left(\int_{0}^{1}\left|\int_{0}^{1} K\left(\gamma(s), \gamma\left(s^{\prime}\right)\right) h\left(s^{\prime}\right) d s^{\prime}\right|^{2} d s\right)^{1 / 2} \leq C \theta^{-1-\varepsilon}\|h\|_{L^{2}([0,1])}
$$

if

$$
K\left(\gamma(s), \gamma\left(s^{\prime}\right)\right)=\int \widetilde{a}(t) e^{-i \lambda t}\left(\left(I-Q_{\theta, \lambda}\right) \circ \widetilde{\beta}(P / \lambda) e^{i t P} \circ\left(I-Q_{\theta, \lambda}\right)^{*}\right)\left(\gamma(s), \gamma\left(s^{\prime}\right)\right) d t .
$$

Choose $\rho \in C_{0}^{\infty}\left(\mathbb{R}^{n}\right)$ now satisfying $\rho(s)=1,|s| \leq 1$, and supp $\rho \subset[-2,2]$. Then, by (5.5), modulo an $O\left(\lambda^{-N}\right)\|h\|_{2}$ error, the left side of (5.7) equals

$$
\left(\int_{0}^{1}\left|\int_{0}^{1} K_{\lambda, \theta}\left(\gamma(s), \gamma\left(s^{\prime}\right)\right) h\left(s^{\prime}\right) d s^{\prime}\right|^{2} d s\right)^{1 / 2}
$$

if

$$
\begin{aligned}
& K_{\lambda, \theta}\left(\gamma(s), \gamma\left(s^{\prime}\right)\right) \\
& =\int \rho\left(\lambda \theta^{2+\varepsilon} t\right) \widetilde{a}(t) e^{-i \lambda t}\left(\left(I-Q_{\theta, \lambda}\right) \circ \widetilde{\beta}(P / \lambda) e^{i t P} \circ\left(I-Q_{\theta, \lambda}\right)^{*}\right)\left(\gamma(s), \gamma\left(s^{\prime}\right)\right) d t .
\end{aligned}
$$

Our assumptions give that $\lambda \theta^{2+\varepsilon} \gg 1$, and so the above integral is just over a small interval of length $\lesssim \lambda^{-1} \theta^{-2-\varepsilon}$. We claim that for parameters as above we have the uniform estimates

$$
\begin{aligned}
& \left|K_{\lambda, \theta}\left(\gamma(s), \gamma\left(s^{\prime}\right)\right)\right| \leq C \lambda^{\frac{1}{2}}\left|s-s^{\prime}\right|^{-\frac{1}{2}}, \\
& \quad \text { if } s, s^{\prime} \in[0,1], \quad \text { and }\left|s-s^{\prime}\right| \leq 10 \lambda^{-1} \theta^{-2-\varepsilon},
\end{aligned}
$$

and

$$
\begin{aligned}
\left|K_{\lambda, \theta}\left(\gamma(s), \gamma\left(s^{\prime}\right)\right)\right| \leq C_{N} \lambda^{-N} & \forall N, \\
& \text { if } \quad s, s^{\prime} \in[0,1], \quad \text { and }\left|s-s^{\prime}\right| \geq 10 \lambda^{-1} \theta^{-2-\varepsilon} .
\end{aligned}
$$

If we had this we would obtain (5.7). For by (5.10) agrees with the left side of (5.7) up to trivial errors, we conclude that, up to such errors, the left side of (5.7) is dominated by

$$
\lambda^{\frac{1}{2}}\left(\int_{0}^{1}\left|\int_{\left|s-s^{\prime}\right| \leq 10 \lambda^{-1} \theta^{-2-\varepsilon}} h\left(s^{\prime}\right)\right| s-\left.\left.s^{\prime}\right|^{-\frac{1}{2}} d s^{\prime}\right|^{2} d s\right)^{1 / 2} \leq C \theta^{-1-\varepsilon}\|h\|_{L^{2}},
$$

as desired, by Young's inequality. 
We shall postpone the proof of 5.10 - 5.11) for the moment since it follows from arguments that we shall use in the proof of Lemma 5.1 .

Proof of Lemma 5.1. We need to prove (5.4) and (5.5). The proof is very similar to the proof of (3-14) in [2].

To prove 5.4, let us first assume that the $x \in \mathcal{T}_{\lambda^{-\frac{1}{2}}}(\gamma)$ lies on our $\gamma \in \Pi$. The kernel in the left side of (5.4) is a Lagrangian distribution in $y$. If we choose geodesic normal coordinates vanishing at $x$ so that $\gamma$ is part of the first coordinate axis then, assuming as we are in (5.4) that $y$ is of distance $\leq 1$ from $x$, modulo $O\left(\lambda^{-N}\right)$ errors, if the $c_{0}$ in $(2.9)$ is sufficiently small, this kernel is of the form

$$
I_{t}(y)=\int_{\mathbb{R}^{n}} e^{-i y \cdot \xi+i t|\xi|} a_{\theta, \lambda}(t, y, \xi) d \xi,
$$

with amplitude satisfying

$$
a_{\theta, \lambda}(t, y, \xi)=0, \quad \text { if }|\xi| \notin[\lambda / 10,10 \lambda], \text { or }\left|\xi^{\prime}\right| /|\xi| \leq 2 B \theta \text {, if } \xi^{\prime}=\left(\xi_{2}, \ldots, \xi_{n}\right),
$$

for some $B$ independent of $\lambda, \theta$, by virtue of 2.8 and 2.10 , as well as

$$
\left|\partial_{t}^{j} D_{\xi}^{\alpha} a_{\theta, \lambda}(t, y, \xi)\right| \leq C_{\alpha, j}(\lambda / \theta)^{-|\alpha|} .
$$

We are using here the fact that if $c_{0}$ in 2.9 is small enough then the symbol of the dyadic operators $\left(I-Q_{\theta, \lambda}\right) \circ \beta(P / \lambda)$ must vanish in a cone of aperture $\approx \theta$ about the $\xi_{1}$-axis because of 2.8 and 2.9$)$ and the fact that the symbol of $\beta(P / \lambda)$ is supported in the region were $|\xi| \approx \lambda$ by (3.18).

We would get (5.4) for $x \in \gamma$ if we could show that

$$
I_{t}(y)=O\left(\lambda^{-N}\right), \quad \text { if } \quad\left|y^{\prime}\right| \leq C_{1} \lambda^{-\frac{1}{2}}, \text { and }|t| \geq C_{0} \lambda^{-\frac{1}{2}} \theta^{-1},
$$

for some large constant $C_{0}$ (independent of $\theta$ and $\lambda$ ) since, in the coordinates we are using, $\left|y^{\prime}\right| \leq C_{1} \lambda^{-\frac{1}{2}}$, is is satisfied with $C_{1}$ fixed by all points in $\mathcal{T}_{\lambda^{-\frac{1}{2}}}(\gamma)$ with coordinates $y$.

To prove this we note that we can obtain favorable lower bounds for the phase functions for the Lagrangian distributions in $\left[5.12\right.$. Specifically, we have for points in $\mathcal{T}_{\lambda^{-\frac{1}{2}}}(\gamma)$ satisfying the first condition in 5.15

$$
\begin{aligned}
\left|\nabla_{\xi^{\prime}}(-y \cdot \xi+t|\xi|)\right|=\left|t \frac{\xi^{\prime}}{|\xi|}-y^{\prime}\right| & \geq\left|t \frac{\xi^{\prime}}{|\xi|}\right|-C_{1} \lambda^{-\frac{1}{2}} \\
& \geq \lambda^{-\frac{1}{2}}, \quad \text { if }\left|\xi^{\prime}\right| /|\xi| \geq B|\theta|, \text { and }|t| \geq C_{0} \lambda^{-\frac{1}{2}} \theta^{-1},
\end{aligned}
$$

provided that $C_{0} B \geq 1$. Using this, by virtue of $(5.13)-(5.14)$, one obtains $(5.15)$ by a simple integration by parts argument. Indeed, by $(5.14)$, each integration by parts yields a gain of $\approx \lambda^{-\frac{1}{2}} / \theta \geq \lambda^{-\delta_{0}}$, since we are assuming that $\theta \geq \lambda^{-\frac{1}{2}+\delta_{0}}$.

Thus, we have proved $\sqrt{5.4}$ when $x$ lies on $\gamma$. The same argument will work for $x \in \mathcal{T}_{\lambda^{-\frac{1}{2}}}(\gamma)$ since $\lambda^{-\frac{1}{2}} \ll \theta$. Given any $x \in \mathcal{T}_{\lambda^{-\frac{1}{2}}}(\gamma)$, pick the geodesic segment $\gamma_{x} \in \Pi$ of closest distance to $\gamma$, using the standard metric on $S^{*} M$ and identifying $\Pi$ with $S^{*} M$. Because of the relationship between $\theta$ and $\lambda^{-\frac{1}{2}}$ the operators $Q_{\theta, \lambda}$ also localize to a $\approx \theta$ neighborhood of $S^{*} \gamma_{x}$, just as it does for $S^{*} \gamma$, due to the fact that the distance between the central geodesic $\gamma$ and any such $\gamma_{x}$ must be $O\left(\lambda^{-\frac{1}{2}}\right)$ when $x \in \mathcal{T}_{\lambda^{-\frac{1}{2}}}(\gamma)$. So if we choose geodesic normal coordinates as above about $x$, we still can write the kernel as a function of the other variables as in (5.12). So the argument for the special case that we 
have just completed implies that the same bounds are valid for all $x \in \mathcal{T}_{\lambda^{-\frac{1}{2}}}(\gamma)$, which completes the proof of (5.4).

The proof of (5.5) is very similar. If we use geodesic normal coordinates about $\gamma(s)$ as above then, modulo trivial errors, the kernels in the left side of 5.5 are of the form

$$
I_{t}\left(s, s^{\prime}\right)=\int_{\mathbb{R}^{2}} e^{-i\left(s-s^{\prime}\right) \xi_{1}+i t|\xi|} a_{\theta, \lambda}\left(t, s, s^{\prime}, \xi\right) d \xi,
$$

with amplitudes satisfying (5.13)-5.14) (with $y$ replaced by $\left(s, s^{\prime}\right)$ ). Clearly if $\left|\xi_{2}\right| /|\xi| \geq$ $B \theta$ and if $|t| \geq \frac{1}{2} \lambda^{-1} \theta^{-2-\varepsilon}$, we have

$$
\left|\frac{\partial}{\partial \xi_{2}}\left(-\left(s-s^{\prime}\right) \xi_{1}+t|\xi|\right)\right| \geq B \lambda^{-1} \theta^{-1-\varepsilon} .
$$

Thus, by $(5.13)$, every time we integrate by parts in $\xi_{2}$ we gain by $\theta^{\varepsilon}=\lambda^{-\delta_{0} \varepsilon}$, and, therefore, since we are assuming that $\delta_{0}$ and $\varepsilon$ are both positive, we have for large $\lambda$

$$
I_{t}\left(s, s^{\prime}\right)=O\left(\lambda^{-N}\right), \quad \text { if }|t| \geq \frac{1}{2} \lambda^{-1} \theta^{-2-\varepsilon},
$$

which means that we have [5.5) with no condition on $s, s^{\prime} \in[0,1]$, if $|t| \geq \frac{1}{2} \lambda^{-1} \theta^{-2-\varepsilon}$. If we integrate by parts in $\xi_{1}$ we find that we also have

$$
I_{t}\left(s, s^{\prime}\right)=O\left(\lambda^{-N}\right), \quad \text { if } \quad|t| \leq \frac{1}{2} \lambda^{-1} \theta^{-2-\varepsilon}, \text { and }\left|s-s^{\prime}\right| \geq \lambda^{-1} \theta^{-2-\varepsilon},
$$

which yields the remaining part of $(5.5)$.

Proof of 5.10 - 5.11). The kernels involved are just

$$
\begin{aligned}
\int \rho\left(\lambda \theta^{2+\varepsilon} t\right) \widetilde{a}(t) e^{-i \lambda t} & I_{t}\left(s, s^{\prime}\right) d t \\
& =\iint \rho\left(\lambda \theta^{2+\varepsilon} t\right) \tilde{a}(t) e^{i\left(s-s^{\prime}\right) \xi_{1}} a_{\theta, \lambda}\left(t, s, s^{\prime}, \xi\right) e^{i t(|\xi|-\lambda)} d t d \xi .
\end{aligned}
$$

In view of the support properties of $\rho$, (5.11) follows from the proof of (5.16), since the latter shows that $I_{t}\left(s, s^{\prime}\right)$ is rapidly decreasing if $\left|s-s^{\prime}\right| \geq 2|t|$ and if $|t| \lesssim \lambda^{-1} \theta^{-2-\varepsilon}$.

To prove (5.10), let us first note that if $\widetilde{K}_{\theta, \lambda}$ denotes the analog of the kernel in 5.9 but without the $\left(I-Q_{\theta, \lambda}\right)$ operators in the left and right, i.e.,

$$
\widetilde{K}_{\theta, \lambda}\left(\gamma(s), \gamma\left(s^{\prime}\right)\right)=\int \rho\left(\lambda \theta^{2+\varepsilon} t\right) \widetilde{a}(t) e^{-i \lambda t}\left(\widetilde{\beta}(P / \lambda) e^{i t P}\right)\left(\gamma(s), \gamma\left(s^{\prime}\right)\right) d t,
$$

then

$$
\left|\widetilde{K}_{\theta, \lambda}\left(\gamma(s), \gamma\left(s^{\prime}\right)\right)\right|=O\left(\lambda^{\frac{1}{2}}\left|s-s^{\prime}\right|^{-\frac{1}{2}}\right) .
$$

To see this we use the Hadamard parametrix and the calculus of Fourier integrals to see that modulo a $O\left(\lambda^{-N}\right)$ error term

$$
\left(\widetilde{\beta}(P / \lambda) e^{i t P}\right)\left(\gamma(s), \gamma\left(s^{\prime}\right)\right)=\int_{\mathbb{R}^{2}} e^{i\left(s-s^{\prime}\right) \xi_{1}+i t|\xi|} \alpha\left(t, s, s^{\prime},|\xi|\right) d \xi,
$$

where $\alpha$ is a zero-order symbol. Thus, modulo trivial errors,

$$
\begin{aligned}
& \widetilde{K}_{\theta, \lambda}\left(\gamma(s), \gamma\left(s^{\prime}\right)\right)= \\
& \qquad \iint_{0}^{\infty} e^{i t(r-\lambda)} \rho\left(\lambda \theta^{2+\varepsilon} t\right) \alpha\left(t, s, s^{\prime}, r\right) r\left(\int_{S^{1}} e^{i r\left(s-s^{\prime}\right)\langle(1,0), \omega\rangle} d \omega\right) d r d t .
\end{aligned}
$$


Integrating by parts in $t$ shows that this expression is majorized by

$$
\begin{array}{rl}
\int_{0}^{\infty}\left(\lambda \theta^{2+\varepsilon}\right)^{-1}\left(1+\lambda^{-1} \theta^{-2-\varepsilon}|r-\lambda|\right)^{-3} & r d r \\
& =\lambda \int_{0}^{\infty} \theta^{-2-\varepsilon}\left(1+\theta^{-2-\varepsilon}|r-1|\right)^{-3} r d r=O(\lambda),
\end{array}
$$

since $\theta^{-2-\varepsilon}>1$. Thus, $\sqrt{5.10^{\prime}}$ is valid when $\left|s-s^{\prime}\right| \leq \lambda^{-1}$. To handle the remaining case, we recall that, by stationary phase,

$$
\int_{S^{1}} e^{i x \cdot \omega} d \omega=O\left(|x|^{-\frac{1}{2}}\right), \quad|x| \geq 1 .
$$

If we plug this into (5.18) with $x=r\left(s-s^{\prime}, 0\right)$, and, as above, integrate by parts in $t$, we conclude that when $\lambda^{-1} \lesssim\left|s-s^{\prime}\right|$ we have

$$
\begin{array}{r}
\left|\widetilde{K}_{\theta, \lambda}\left(\gamma(s), \gamma\left(s^{\prime}\right)\right)\right| \lesssim \int_{0}^{\infty}\left(\lambda \theta^{2+\varepsilon}\right)^{-1}\left(1+\lambda^{-1} \theta^{-2-\varepsilon}|r-\lambda|\right)^{-3}\left(r\left|s-s^{\prime}\right|\right)^{-\frac{1}{2}} r d r \\
=O\left(\lambda^{\frac{1}{2}}\left|s-s^{\prime}\right|^{-\frac{1}{2}}\right),
\end{array}
$$

as claimed, which finishes the proof of $\left(5.10^{\prime}\right)$.

Next, since the symbol of $Q_{\theta, \lambda}$ is as in (2.8), it follows that if the constant $c_{0}>0$ in 2.9 is small enough then $Q_{\theta, \lambda} \circ \widetilde{\beta}(P / \lambda)$ and $\widetilde{\beta}(P / \lambda) \circ Q_{\theta, \lambda}^{*}$ both have symbols supported in the set where $|\xi| \approx \lambda$ and $\left|\xi_{1}\right| /|\xi| \leq C \theta$ for some constant $C$. Thus, it follows that $K_{\theta, \lambda}-\widetilde{K}_{\theta, \lambda}$ can be written in the form 5.17) except with $I_{t}\left(s, s^{\prime}\right)$ replaced by

$$
\widetilde{I}_{t}\left(s, s^{\prime}\right)=\int_{\mathbb{R}^{2}} e^{i\left(s-s^{\prime}\right) \xi_{1}+i t|\xi|} \widetilde{a}_{\theta, \lambda}\left(t, s, s^{\prime}, \xi\right) d \xi
$$

where the amplitude here satisfies the bounds in $(5.14)$, but in place of $(5.13)$, we have

$$
\widetilde{a}_{\theta, \lambda}\left(t, s, s^{\prime}, \xi\right)=0 \text { if }|\xi| \notin[\lambda / 10,10 \lambda] \text { or }\left|\xi_{2}\right| /|\xi| \geq C \theta
$$

From this, and straighforward analogs of the above calculations, we deduce that, modulo trivial errors,

$$
\begin{aligned}
& \mid K_{\theta, \lambda}\left(\left(\gamma(s), \gamma\left(s^{\prime}\right)\right)-\widetilde{K}_{\theta, \lambda}\left(\left(\gamma(s), \gamma\left(s^{\prime}\right)\right) \mid\right.\right. \\
& \quad \lesssim\left(\lambda \theta^{2+\varepsilon}\right)^{-1} \int_{\left\{\xi \in \mathbb{R}^{2}:\left|\xi_{2}\right| \leq C \theta|\xi|\right\}}\left(1+\lambda^{-1} \theta^{-2-\varepsilon}|| \xi|-\lambda|\right)^{-3} d \xi=O(\lambda \theta) .
\end{aligned}
$$

As $\lambda \theta \leq \lambda^{\frac{1}{2}}\left|s-s^{\prime}\right|^{-\frac{1}{2}}$ if $\left|s-s^{\prime}\right| \leq \lambda^{-1} \theta^{-2}$, by (5.10'), it suffices to show that

$$
\begin{aligned}
\iint_{\mathbb{R}^{2}} \rho\left(\lambda \theta^{2+\varepsilon} t\right) e^{i t(|\xi|-\lambda)} e^{i\left(s-s^{\prime}\right) \xi_{1}} \widetilde{a}_{\theta, \lambda}\left(t, s, s^{\prime}, \xi\right) d \xi d t & \\
& =O\left(\lambda^{\frac{1}{2}}\left|s-s^{\prime}\right|^{-\frac{1}{2}}\right), \quad \text { if }\left|s-s^{\prime}\right| \geq \lambda^{-1} \theta^{-2} .
\end{aligned}
$$

But this follows from the argument that we just used for $\widetilde{K}_{\theta, \lambda}$ if, instead of $[5.19$, we use the fact that for each fixed $j=0,1, \ldots$,

$$
\int_{S^{1}} e^{i r x \cdot \omega} \partial_{t}^{j} \widetilde{a}_{\theta, \lambda}\left(t, s, s^{\prime}, r \omega\right) d \omega=O\left((\lambda|x|)^{-\frac{1}{2}}\right), \quad \text { if } r \approx \lambda, \quad \text { and } \quad \lambda^{-1} \theta^{-2} \lesssim|x|,
$$

due to 5.13 and (5.14). Since the $\widetilde{a}_{\theta, \lambda}$ satisfy the bounds in 5.14, this follows from the method of stationary phase by slightly modifying the standard proof of 5.19 . 


\section{ACKNOWLEDGEMENTS}

With pleasure, we thank William Minicozzi and Steve Zelditch for many helpful discussions and for generously sharing their knowledge.

\section{REFERENCES}

[1] Bérard, P., On the wave equation on a compact Riemannian manifold without conjugate points, Math. Z. 155 (1977), 249-276.

[2] Blair, M. D. and Sogge, C. D., Refined and microlocal Kakeya-Nikodym bounds for eigenfunctions in two dimensions, Anal. PDE 8 (2015), 747-764.

[3] Blair, M. D. and Sogge, C. D., On Kakeya-Nikodym averages, $L^{p}$-norms and lower bounds for nodal sets of eigenfunctions in higher dimensions, J. European Math. Soc., 17 (2015), 2513-2543.

[4] Blair, M. D. and Sogge, C. D., Refined and microlocal Kakeya-Nikodym bounds of eigenfunctions in higher dimensions, preprint.

[5] Bourgain, J., Geodesic restrictions and $L^{p}$-estimates for eigenfunctions of Riemannian surfaces, "Linear and complex analysis," 27-35, Amer. Math. Soc. Transl. Ser. 2, 226, Amer. Math. Soc., Providence, RI, 2009.

[6] Burq, N., Gérard, P. and Tzvetkov, N., Restrictions of the Laplace-Beltrami eigenfunctions to submanifolds, Duke Math. J. 138 (2007), 445-486.

[7] Chavel, I., Riemannian geometry, A modern introduction, Second edition, Cambridge Studies in Advanced Mathematics, 98. Cambridge University Press, Cambridge, 2006.

[8] Cheeger, J. and Ebin, D. G., Comparison theorems in Riemannian geometry, Revised reprint of the 1975 original, AMS Chelsea Publishing, Providence, RI, 2008.

[9] Chen, X., An improvement on eigenfunction restriction estimates for compact boundaryless Riemannian manifolds with nonpositive sectional curvature, Trans. Amer. Math. Soc. 367 (2015), 4019-4039.

[10] Chen, X. and Sogge, C. D., A few endpoint geodesic restriction estimates for eigenfunctions, Comm. Math. Phys. 329 (2014), 435-459.

[11] Colding, T. H. and Minicozzi, W. P., II, Lower bounds for nodal sets of eigenfunctions, Comm. Math. Phys. 306 (2011), 777-784.

[12] Donnelly, H.; Fefferman, C. Nodal sets of eigenfunctions on Riemannian manifolds, Invent. Math. 93 (1988), 161-183.

[13] Greenleaf, A. and Seeger, A., Fourier integral operators with fold singularities, J. Reine Angew. Math. 455 (1994), 35-56.

[14] Han, X., Small scale quantum ergodicity on negatively curved manifolds, arXiv:1410.3911 (2014).

[15] Hassell, A. and Tacy, M., Improvement of eigenfunction estimates on manifolds of nonpositive curvature, Forum Math. 27 (2015), 1435-1451.

[16] Hezari, H. and Rivière, G., $L^{p}$ norms, nodal sets, and quantum ergodicity, arXiv:1411.4078 (2014).

[17] Hezari, H. and Sogge, C. D., A natural lower bound for the size of nodal sets, Anal. PDE 5 (2012), $1133-1137$.

[18] Ralston, J. Approximate eigenfunctions of the Laplacian, J. Differential Geometry 12 (1977), $87-100$.

[19] Sogge, C. D., Concerning the $L^{p}$ norm of spectral clusters for second-order elliptic operators on compact manifolds, J. Funct. Anal. 77 (1988), 123-138.

[20] Sogge, C. D., Fourier integrals in classical analysis, Cambridge Tracts in Mathematics, 105 Cambridge University Press, Cambridge, 1993.

[21] Sogge, C. D., Kakeya-Nikodym averages and $L^{p}$-norms of eigenfunctions, Tohoku Math. J. 63 (2011), 519-538.

[22] Sogge, C. D., Localized $L^{p}$-estimates of eigenfunctions: A note on an article of Hezari and Rivière, arXiv: 1503.07238

[23] Sogge, C. D., Toth, J. A. and Zelditch, S., About the blowup of quasi-modes on Riemannian manifolds, J. Geom. Anal. 21 (2011), 150-173.

[24] Sogge, C. D. and Zelditch, S., Riemannian manifolds with maximal eigenfunction growth, Duke Math. J. 114 (2002), 387-437. 
[25] Sogge, C. D. and Zelditch, S., Lower bounds on the Hausdorff measure of nodal sets Math. Res. Lett. 18 (2011), 25-37.

[26] Lower bounds on the Hausdorff measure of nodal sets II, Math. Res. Lett. 19 (2012), 1361-1364.

[27] Sogge, C. D. and Zelditch, S., On eigenfunction restriction estimates and $L^{4}$-bounds for compact surfaces with nonpositive curvature, "Advances in analysis: the legacy of Elias M. Stein", 447-461, Princeton Math. Ser., 50, Princeton Univ. Press, Princeton, NJ, 2014.

[28] Sogge, C. D. and Zelditch, S., Focal points and sup-norms of eigenfunctions, Rev. Mat. Iberoamericana, to appear.

[29] Sogge, C. D. and Zelditch, S., Focal points and sup-norms of eigenfunctions manifolds II: the two-dimensional case, Rev. Mat. Iberoamericana, to appear.

[30] Sogge, C. D. and Zelditch, S., A note on the $L^{p}$ norms of quasi-modes, arXiv:1401.0345

[31] Tataru, Daniel, On the regularity of boundary traces for the wave equation, Ann. Scuola Norm. Sup. Pisa Cl. Sci. 26 (1998), 185-206.

[32] Toponogov, V. A., Riemann spaces with curvature bounded below, (Russian), Uspehi Mat. Nauk 14 (1959), 87-130.

[33] Yau, S.T. ,Survey on partial differential equations in differential geometry. Seminar on Differential Geometry, pp. 3-71, Ann. of Math. Stud., 102, Princeton Univ. Press, Princeton, N.J., 1982.

Department of Mathematics and Statistics, University of New Mexico, Albuquerque, NM 87131, USA

E-mail address: blair@math.unm.edu

Department of Mathematics, Johns Hopkins University, Baltimore, MD 21093, USA

E-mail address: sogge@jhu.edu 